Pacific

Journal of

Mathematics

MASLOV TYPE INDEX THEORY FOR LINEAR HAMILTONIAN SYSTEMS WITH BOLZA BOUNDARY VALUE CONDITIONS AND MULTIPLE SOLUTIONS FOR NONLINEAR HAMILTONIAN SYSTEMS

YUJUN DONG

Volume $221 \quad$ No. 2

October 2005 


\title{
MASLOV TYPE INDEX THEORY FOR LINEAR HAMILTONIAN SYSTEMS WITH BOLZA BOUNDARY VALUE CONDITIONS AND MULTIPLE SOLUTIONS FOR NONLINEAR HAMILTONIAN SYSTEMS
}

\author{
YUJUN DONG \\ In memory of my father
}

\begin{abstract}
We study the classification of linear Hamiltonian systems satisfying Bolza boundary conditions and its applications to nonlinear Hamiltonian systems.
\end{abstract}

\section{Introduction}

Ivar Ekeland [1990] discussed the classification of convex Hamiltonian systems of the form

$$
\dot{x}=J B(t) x, \quad x(0)=x(\tau),
$$

where

$$
J=\left(\begin{array}{rr}
0 & -I_{n} \\
I_{n} & 0
\end{array}\right)
$$

$I_{n}$ being the $n \times n$ identity matrix. That is, for any continuous symmetric positive definite matrix map $B:[0, \tau] \rightarrow \mathrm{GL}(2 n)$ with $B(0)=B(\tau)$, he defined a pair of numbers $\left(i^{E}(B), v^{E}(B)\right) \in\{0,1,2, \ldots\} \times\{0,1,2, \ldots, 2 n\}$ by making use of dual variational methods. This pair of integers is called the Ekeland index of $B$.

We define, as usual,

$$
\begin{aligned}
& \operatorname{Sp}(2 n)=\left\{M \in \operatorname{GL}(2 n, \mathbb{R}) \mid M^{T} J M=J\right\}, \\
& \mathscr{P}_{\tau}(2 n)=\left\{\gamma: C([0, \tau], \operatorname{Sp}(2 n)) \mid \gamma(0)=I_{2 n}\right\} .
\end{aligned}
$$

For any $\gamma \in \mathscr{P}_{\tau}(2 n)$, the Maslov-type index of $\gamma$ is defined to be a pair of integers $\left(i^{L}(\gamma), v^{L}(\gamma)\right) \in \mathbb{Z} \times\{0,1,2, \ldots, 2 n\}$; see [Conley and Zehnder 1984; Long and

\section{MSC2000: 34B15.}

Keywords: Hamiltonian system, Bolza problem, existence and multiplicity of solutions, Maslov

type index theory, relative Morse index, $\mu$-index, Leray-Schauder degree theory, dual variational principle, Morse theory.

Partially supported by the National Natural Science Foundation of China (10251001), the Educational Committee Foundation of Jiangsu, and the Natural Science Foundation of Jiangsu (BK2002023). 
Zehnder 1990; Long 1990; Long 1997]. Denote by $\gamma_{B}(t)$ the matrizant of (1-1), that is, the solution of

$$
\dot{\gamma}_{B}(t)=J B(t) \gamma_{B}(t), \quad \gamma_{B}(0)=I_{2 n} .
$$

Then $\gamma_{B} \in \mathscr{P}_{\tau}(2 n)$ and

$$
i^{L}\left(\gamma_{B}\right)=i^{E}(B)+n, \quad v^{L}\left(\gamma_{B}\right)=v^{E}(B),
$$

whenever $B$ is positive definite. So the Maslov-type index is more general than the Ekeland index. These index theories (about the properties of the index) and their iteration theories have important applications in the study of nonlinear Hamiltonian systems. See [Fei 1995; Conley and Zehnder 1984; Long and Zehnder 1990; Fei and Qiu 1997; Chang et al. 1997; Su 1998; Li and Liu 1989] for multiple periodic solutions of asymptotically linear Hamiltonian systems, [Ekeland and Hofer 1985; Dong and Long 1997] for Rabinowitz's minimal periodic problem, and [Ekeland and Hofer 1987; Long and Zhu 2002; Liu et al. 2002] for multiple closed characteristics on compact convex hyper-surfaces in $\mathbb{R}^{2 n}$. For a systematic treatment and other applications, one can refer to the excellent books [Ekeland 1990; Long 2002].

Let $\mathscr{L}_{2 n}^{\infty}:=\left\{B:(0,1) \rightarrow \mathrm{GL}(2 n) \mid B(t)=\left(b_{i j}(t)\right)_{2 n \times 2 n}, b_{i j} \in L^{\infty}(0,1)\right.$ and $b_{i j}=b_{j i}$ for all $\left.i, j=1,2, \ldots, 2 n\right\}$. Throughout this paper, for any two symmetric matrices $A_{1}$ and $A_{2}$, we write $A_{1} \leq A_{2}$ if $A_{2}-A_{1}$ is positive semidefinite, and write $A_{1}<A_{2}$ if $A_{2}-A_{1}$ is positive definite. For any $B_{1}, B_{2} \in \mathscr{L}_{2 n}^{\infty}$, we write $B_{1} \leq B_{2}$ if $B_{1}(t) \leq B_{2}(t)$ for a.e. $t \in(0,1)$; write $B_{1}<B_{2}$ if $B_{1} \leq B_{2}$ and $B_{1}(t)<B_{2}(t)$ on a subset of $(0,1)$ with positive measure. In this paper we consider the classification of systems of the form

$$
\dot{x}=J B(t) x, \quad P x(0)=0=P x(1),
$$

where $B \in \mathscr{L}_{2 n}^{\infty}$ and $P x=\left(x_{1}, \ldots, x_{n}\right)^{T} \in \mathbb{R}^{n}$ for any $x=\left(x_{1}, \ldots, x_{2 n}\right)^{T} \in \mathbb{R}^{2 n}$. Specifically, to any $B \in \mathscr{L}_{2 n}^{\infty}$ we associate a pair of numbers $(i(B), v(B)) \in \mathbb{Z} \times$ $\{0,1, \cdots, n\}$. The nullity $v(B)$ is the dimension of the solution space of (1-2). To define the index $i(B)$ we proceed in two steps. At first, we give the definition of $i\left(\lambda I_{2 n}\right)$ for any $\lambda \in \mathbb{R}$. Second, for any two $B_{1}, B_{2} \in \mathscr{L}_{2 n}^{\infty}$ with $B_{1}<B_{2}$ we consider the nullity $v\left((1-\lambda) B_{1}+\lambda B_{2}\right)$. We prove that the number of $\lambda \in[0,1)$ with $v\left((1-\lambda) B_{1}+\lambda B_{2}\right) \neq 0$ is finite and define the relative Morse index as $I\left(B_{1}, B_{2}\right)=$ $\sum_{\lambda \in[0,1)} v\left((1-\lambda) B_{1}+\lambda B_{2}\right)$. We prove the relative Morse index satisfies additivity properties and that $i\left(\lambda I_{2 n}\right)-I\left(B, \lambda I_{2 n}\right)$ is independent of $\lambda \in \mathbb{R}$ with $\lambda I_{2 n}>B$; we define $i(B)$ to be this number. We define an Ekeland type index $i_{\mu}(B)$, called the $\mu$-index of $B$ and satisfying $I\left(B_{1}, B_{2}\right)=i_{\mu}\left(B_{2}\right)-i_{\mu}\left(B_{1}\right)$. In this way we can prove additivity for the relative Morse index. This is the content of Section 2. In the process we only use the spectral theory of self-adjoint compact operators. 
In [Dong 2005] we discussed the classification of the second-order Hamiltonian system

$$
x^{\prime \prime}+A(t) x=0, \quad x(0)=0=x(1) .
$$

Namely, to every $A \in \mathscr{L}_{n}^{\infty}$ we associated a pair of numbers $\left(m^{0}(A), m^{-}(A)\right)$. By making the change $z_{1}=x, z_{2}=\dot{x}, z=\left(z_{1}, z_{2}\right)$, we see that (1-3) is a special case of (1-2) with $B$ the block-diagonal matrix $\operatorname{diag}\left\{A, I_{n}\right\}$. At the end of Section 2, we prove that $m^{0}(A)=v\left(\operatorname{diag}\left\{A, I_{n}\right\}\right)$ and that $m^{-}(A)=i\left(\operatorname{diag}\left\{A, I_{n}\right\}\right)$.

In Section 3 we discuss multiple solutions for the system

$$
\dot{x}=J H^{\prime}(t, x), \quad P x(0)=0=P x(1) .
$$

We first use the Leray-Schauder degree theory to obtain the existence of solutions and of nontrivial solutions. Then we use the dual variational method and Morse theory to discuss the multiple solutions of (1-4). The index and $\mu$-index play an important role in the discussion. We stress that by making use of the $\mu$-index, dual variational methods can be used instead of the saddle point reduction method whenever $H^{\prime \prime}(t, x)$ is bounded. Ekeland [1990] discussed the problem (1-4) and called it the Bolza problem. The method used here is also suitable for Hamiltonian systems with periodic boundary value conditions, for which we will write another paper.

\section{Maslov type index theory for linear Hamiltonian systems satisfying Bolza boundary value conditions}

As in the introduction we set $P x=\left(x_{1}, \ldots, x_{n}\right)^{T} \in \mathbb{R}^{n}$ for any $x=\left(x_{1}, \ldots, x_{2 n}\right)^{T} \in$ $\mathbb{R}^{2 n}$. We shall give a classification for the system

$$
\dot{x}=J B(t) x \text { for } t \in(0,1), \quad P x(0)=0=P x(1) ;
$$

that is, for any $B \in \mathscr{L}_{2 n}^{\infty}$, we shall define $i(B) \in \mathbb{Z}$ and $v(B) \in\{0,1, \ldots, n\}$, called the index and nullity of $B$ respectively. We begin with the nullity. Set

$$
W:=\left\{x:[0,1] \rightarrow \mathbb{R}^{2 n} \mid x \text { continuous on }[0,1], \operatorname{Px}(0)=0=\operatorname{Px}(1), \dot{x} \in L^{2}\right\},
$$

(where $L^{2}:=L^{2}\left((0,1) ; \mathbb{R}^{2 n}\right)$ ), with the norm

$$
\|x\|_{W}:=\left(\int_{0}^{1}\left(|\dot{x}(t)|^{2}+|x(t)|^{2}\right) d t\right)^{1 / 2},
$$

where $|x|=\left(\sum_{i=1}^{2 n}\left|x_{i}\right|\right)^{1 / 2}$ for $x=\left(x_{1}, \ldots, x_{2 n}\right)^{T} \in \mathbb{R}^{2 n}$. Also define $\Lambda_{1}: W \subset$ $L^{2} \rightarrow L^{2}$ by $\left(\Lambda_{1} x\right)(t):=J \dot{x}(t)$ and $\bar{B}: L^{2} \rightarrow L^{2}$ by $(\bar{B} x)(t)=B(t) x(t)$. Then $\Lambda_{1}$ and $\bar{B}$ are self-adjoint operators and $\bar{B}$ is bounded. 
Definition 2.1. For any $B \in \mathscr{L}_{2 n}^{\infty}$, the nullity of $B$ is defined as

$$
v(B)=\operatorname{dim} \operatorname{ker}\left(\Lambda_{1}+\bar{B}\right) .
$$

By definition, $v(B) \geq 0$. Moreover, $v(B)=0$ if and only if $\operatorname{ker}\left(\Lambda_{1}+\bar{B}\right)=\{\theta\}$, and if and only if the problem (2-1) has no nontrivial solutions $x \neq \theta$.

Let $\gamma(t)$ be the matrizant of $(2-1)$, i.e., the solution of

$$
\dot{\gamma}(t)=J B(t) \gamma(t) \text { for a.e. } t \in(0,1), \quad \gamma(0)=I_{2 n} .
$$

Then

$\operatorname{ker}\left(\Lambda_{1}+\bar{B}\right)=\left\{x=\gamma(t)\left(\begin{array}{l}0 \\ c\end{array}\right) \mid c \in \mathbb{R}^{n}, P x(1)=0\right\} \cong\left\{c \in \mathbb{R}^{n} \mid P \gamma(1)\left(\begin{array}{l}0 \\ c\end{array}\right)=0\right\} \subset \mathbb{R}^{n}$.

Thus $\operatorname{dim} \operatorname{ker}\left(\Lambda_{1}+\bar{B}\right) \leq n$, and $v(B)=\operatorname{dim} \operatorname{ker}\left(\Lambda_{1}+\bar{B}\right) \in\{0,1, \ldots, n\}$. In particular, if $B \equiv \lambda I_{2 n}$, we have

$\gamma(t)=\exp J \lambda t=\left(\begin{array}{rr}I_{n} \cos \lambda t & -I_{n} \sin \lambda t \\ I_{n} \sin \lambda t & I_{n} \cos \lambda t\end{array}\right), P \gamma(1)\left(\begin{array}{l}0 \\ c\end{array}\right)=P\left(\begin{array}{r}-c \sin \lambda \\ c \cos \lambda\end{array}\right)=-c \sin \lambda$,

so $\operatorname{ker}\left(\Lambda_{1}+\bar{B}\right)=\left\{c \in \mathbb{R}^{n} \mid c \sin \lambda=0\right\}$. Therefore, $v\left(\lambda I_{2 n}\right)=0$ if and only if $\lambda / \pi \notin \mathbb{Z}$, and $v\left(\lambda I_{2 n}\right)=n$ if $\lambda / \pi \in \mathbb{Z}$.

Still in the case $B \equiv \lambda I_{2 n}$, the system (2-1) is equivalent to

$$
y^{\prime \prime}+\lambda^{2} y=0 \text { for } t \in(0,1), \quad y(0)=0=y(1),
$$

via the change $y=P x$. For the general second-order Hamiltonian system

$$
x^{\prime \prime}+A(t) x=0 \text { for } t \in(0,1), \quad x(0)=0=x(1),
$$

with any $A \in \mathscr{L}_{n}^{\infty}$, we define as in [Dong 2005]

$$
\psi_{A}(x, x):=\int_{0}^{1}\left(|\dot{x}(t)|^{2}-(A(t) x(t), x(t))\right) d t,
$$

where $x \in F:=\left\{x \in W^{1,2}\left([0,1] ; \mathbb{R}^{n}\right) \mid x(0)=0=x(1)\right\}$. Then we have a $\psi_{A^{-}}$ orthogonal decomposition $F=F^{+}(A) \oplus F^{0}(A) \oplus F^{-}(A)$, and we define

$$
m^{-}(A):=\operatorname{dim} F^{-}(A), \quad m^{0}(A):=\operatorname{dim} F^{0}(A) .
$$

In particular, $m^{0}\left(\lambda^{2} I_{n}\right)=0$ if $\lambda / \pi \notin \mathbb{Z}$, and $m^{0}\left(\lambda^{2} I_{n}\right)=n$ if $\lambda / \pi \in \mathbb{Z}$. Thus $m^{0}\left(\lambda^{2} I_{n}\right)=v\left(\lambda I_{2 n}\right)$ for $\lambda>0$. Letting $x_{1}=y, x_{2}=-\dot{y},(2-3)$ is also equivalent to (2-1) with $B=\operatorname{diag}\left\{\lambda^{2} I_{n}, I_{n}\right\}$. By Definition 2.1, we obtain $v\left(\operatorname{diag}\left\{\lambda^{2} I_{n}, I_{n}\right\}\right)=$ $m^{0}\left(\lambda^{2} I_{n}\right)$.

Proposition 2.2. For any $A \in \mathscr{L}_{n}^{\infty}$,

$$
v\left(\operatorname{diag}\left\{A, I_{n}\right\}\right)=m^{0}(A) .
$$


Proof. Making the change $x_{1}=y, x_{2}=-\dot{y}$, we see that (2-4) is equivalent to (2-1) with $B=\operatorname{diag}\left\{A, I_{n}\right\}$.

In the following we consider the definition of $i(B)$ for any $B \in \mathscr{L}_{2 n}^{\infty}$. Since (2-4) is a special case of (2-1), we begin with $B=\lambda I_{2 n}$. Following [Ekeland 1990], we define $E[\alpha]$ for $\alpha$ real as the integer $a \in \mathbb{Z}$ such that $a<\alpha \leq a+1$; in particular $E[\alpha]=\alpha-1$ for integer $\alpha$. Since (2-3) is equivalent to (2-1) when $B=\lambda I_{2 n}$, and $m^{-}\left(\lambda^{2} I_{n}\right)=n E[\lambda / \pi]$ as $\lambda>0$, we have:

Definition 2.3. For any $\lambda \in \mathbb{R}$, we define

$$
i\left(\lambda I_{2 n}\right)=n E[\lambda / \pi]
$$

In order to define $i(B)$ for any $B \in \mathscr{L}_{2 n}^{\infty}$, we need to compare $B$ with $\lambda I_{2 n}$ for any $\lambda>0$ with $\lambda I_{2 n}>B$.

Definition 2.4. For any $B_{1}, B_{2} \in \mathscr{L}_{2 n}^{\infty}$ with $B_{1}<B_{2}$, set

$$
I\left(B_{1}, B_{2}\right)=\sum_{\lambda \in[0,1)} v\left((1-\lambda) B_{1}+\lambda B_{2}\right) .
$$

We call $I\left(B_{1}, B_{2}\right)$ the relative Morse index, following [Fei 1995; Zhu and Long 1999; Long and Zhu 2000; Long 2002].

In Proposition 2.10 we will show that the relative Morse index is finite.

Proposition 2.5. Assume $B, C \in \mathscr{L}_{2 n}^{\infty}$ with $v(B)=0, C \geq \epsilon I_{2 n}$ for some positive constant $\epsilon \in \mathbb{R}$.

(1) The operator $\Lambda_{1}+\bar{B}: W \rightarrow L^{2}$ is invertible and the inverse $\left(\Lambda_{1}+\bar{B}\right)^{-1}$ : $L^{2} \rightarrow L^{2}$ is compact and self-adjoint.

(2) There exists a sequence $\left\{\lambda_{j}\right\}_{j=-\infty}^{+\infty} \subset \mathbb{R}$ with $\lambda_{j} \rightarrow \pm \infty$ as $j \rightarrow \pm \infty$, such that the problem

$$
J \dot{x}(t)+B(t) x(t)=\lambda_{j} C(t) x(t), \quad P x(0)=0=P x(1)
$$

has a nontrivial solution subspace $E_{j}$ with $\operatorname{dim} E_{j} \leq n$ and $L^{2}=\bigoplus_{j=-\infty}^{+\infty} E_{j}$.

Proof. (1) Let $\gamma(t)$ be the matrizant of (2-1) (see (2-2)). To prove that $\Lambda_{1}+\bar{B}$ is invertible for any $u \in L^{2}$, we have to solve the problem

$$
J \dot{x}+B(t) x=u, \quad P x(0)=0=P x(1) .
$$

The first equation has a general solution

$$
x(t)=\gamma(t) x(0)-\gamma(t) \int_{0}^{t} \gamma(s)^{-1} J u(s) d s .
$$


The second condition gives

$$
x(0)=\left(\begin{array}{l}
0 \\
c
\end{array}\right), P x(1)=P \gamma(1)\left(\begin{array}{l}
0 \\
c
\end{array}\right)-P \gamma(1) \int_{0}^{1} \gamma(s)^{-1} J u(s) d s=0 .
$$

Since $v(B)=0$, setting $\gamma(1)=\left(\begin{array}{ll}\gamma_{11}(1) & \gamma_{12}(1) \\ \gamma_{21}(1) & \gamma_{22}(1)\end{array}\right)$, we see that the equation

$$
0=P \gamma(1)\left(\begin{array}{l}
0 \\
c
\end{array}\right)=\gamma_{12}(1) c
$$

has no nontrivial solutions, so $\gamma_{12}(1)$ is invertible. From (2-6) we have $\Lambda_{1}+\bar{B}$ is invertible and

$$
\left(\left(\Lambda_{1}+\bar{B}\right)^{-1} u\right)(0)=\gamma_{12}(1)^{-1} P \gamma(1) \int_{0}^{1} \gamma(s)^{-1} J u(s) d s .
$$

From (2-5) and the Ascoli-Arzelà theorem, the operator $\left(\Lambda_{1}+\bar{B}\right)^{-1}: L^{2} \rightarrow$ $C\left([0,1], \mathbb{R}^{2 n}\right)$ is compact, and so is the operator $\left(\Lambda_{1}+\bar{B}\right)^{-1}: L^{2} \rightarrow L^{2}$.

(2) Let $(\Pi u)(t)=\left(\Lambda_{1}+\bar{B}\right)^{-1} C(t) u(t)$ for any $u \in L^{2}$. With $(x, y)=\sum_{i=1}^{2 n} x_{i} y_{i}$, the inner product $(u, v)_{1}:=\int_{0}^{1}(C(t) u(t), v(t)) d t$ defines a Hilbert space structure, and

$$
\begin{aligned}
(\Pi u, v)_{1} & =\int_{0}^{1}\left(C(t)\left(\Lambda_{1}+\bar{B}\right)^{-1} C(t) u(t), v(t)\right) d t \\
& =\int_{0}^{1}\left(C(t) u(t),\left(\Lambda_{1}+\bar{B}\right)^{-1} C(t) v(t)\right) d t=(u, \Pi v)_{1}
\end{aligned}
$$

for every $u, v \in L^{2}$. Thus $\Pi: L^{2} \rightarrow L^{2}$ is self-adjoint and compact. By spectral theory, there are $\left\{\mu_{j}\right\}_{j=1}^{\infty} \subset \mathbb{R}$ with $\mu_{j} \rightarrow 0$ as $j \rightarrow \infty$ and a basis $\left\{u_{j}\right\}_{j=1}^{\infty}$ of $L^{2}$ such that

$$
\left(u_{j}, u_{i}\right)_{1}=\delta_{i j}, \Pi u_{j}=\mu_{j} u_{j}, u_{j} \neq \theta .
$$

By the definition of $\Pi$, we have $\left(\Lambda_{1}+\bar{B}\right) \mu_{j} u_{j}=C(t) u_{j}$. So $\mu_{j} \neq 0$ and

$$
J \dot{u}_{j}(t)+B(t) u_{j}(t)=\frac{1}{\mu_{j}} C(t) u_{j}(t), P u_{j}(0)=0=P u_{j}(1) .
$$

Hence, setting $E_{j}:=\operatorname{ker}\left(\Pi-\mu_{j}\right)$, we get

$$
\operatorname{dim} E_{j}=v\left(B-\frac{1}{\mu_{j}} C\right) \leq n .
$$

Letting $\lambda_{j}:=1 / \mu_{j}$, we only need to show that $\lambda_{j}$ is bounded above and below. In fact, assume $\lambda_{j} \geq \bar{\lambda}$ for some $\bar{\lambda} \in \mathbb{R}$. For any $x \in W$, set $C(t) u(t):=J \dot{x}(t)+$ 
$B(t) x(t)$; then $u(t)=\sum_{j=1}^{+\infty} u_{j}(t)$ with $u_{j} \in E_{j}, x(t)=(\Pi u)(t)=\sum_{j=1}^{+\infty} \mu_{j} u_{j}(t)$ and

$$
\begin{aligned}
\int_{0}^{1}(J \dot{x}(t)+B(t) x(t), x(t)) d t & =\int_{0}^{1}(C(t) u(t), x(t)) d t \\
& =\int_{0}^{1} \sum_{i=1}^{\infty} \mu_{i}\left(C(t) u_{i}(t), u_{i}(t)\right) d t \\
& \geq \bar{\lambda} \int_{0}^{1}(C(t) x(t), x(t)) d t
\end{aligned}
$$

Thus there exists a constant $c_{1}$ depending only on $B, C$ and $\bar{\lambda}$ such that

$$
\int_{0}^{1}(J \dot{x}(t), x(t)) d t \geq c_{1}\|x\|_{L^{2}}^{2} \quad \text { for all } x \in W .
$$

As in [Ekeland 1990], set $x_{p}(t):=\exp (p \pi(-J)(t))\left(\begin{array}{l}0 \\ \xi\end{array}\right)$ with $p \in \mathbb{Z}$ and $\xi \in \mathbb{R}^{n}$ satisfying $|\xi|=1$. Then $x_{p}(t)=\left(\begin{array}{c}\xi \sin p \pi t \\ \xi \cos p \pi t\end{array}\right) \in W$, and

$$
\left\|x_{p}\right\|_{L^{2}}^{2}=1, \quad \int_{0}^{1}\left(J \dot{x}_{p}(t), x_{p}(t)\right) d t=p .
$$

This contradicts (2-7) when $p<c_{1}$.

Remark. From this Proposition, when $v\left(B_{1}\right)=0, B_{2}-B_{1} \geq \epsilon I_{2 n}$, the number of $\lambda \in[0,1)$ such that $v\left((1-\lambda) B_{1}+\lambda B_{2}\right) \neq 0$ is finite, and $I\left(B_{1}, B_{2}\right)<+\infty$. According to Proposition 2.10 below, the relative Morse index is finite in general case.

Let card denote the cardinality of a set. When $\lambda_{1}<\lambda_{2}$, by the definition, we have $I\left(\lambda_{1} I_{2 n}, \lambda_{2} I_{2 n}\right)=n \operatorname{card}\left\{j \in \mathbb{Z} \mid j \in\left[\lambda_{1} / \pi, \lambda_{2} / \pi\right)\right\}$, and $I\left(\lambda_{1} I_{2 n}, \lambda_{3} I_{2 n}\right)=$ $I\left(\lambda_{1} I_{2 n}, \lambda_{2} I_{2 n}\right)+I\left(\lambda_{2} I_{2 n}, \lambda_{3} I_{2 n}\right)$ if $\lambda_{3}>\lambda_{2}>\lambda_{1}$. More generally:

Proposition 2.6. Assume $B_{i}, i=1,2,3, \in \mathscr{L}_{2 n}^{\infty}$ with $B_{1}<B_{2}<B_{3}$. Then

$$
I\left(B_{1}, B_{3}\right)=I\left(B_{1}, B_{2}\right)+I\left(B_{2}, B_{3}\right) .
$$

We postpone the proof and first define $i(B)$. From Definition 2.3, for any $\lambda<\lambda_{1}$ we have $i\left(\lambda_{1} I_{2 n}\right)=i\left(\lambda I_{2 n}\right)+I\left(\lambda I_{2 n}, \lambda_{1} I_{2 n}\right)$, so

$$
\begin{aligned}
i\left(\lambda_{1} I_{2 n}\right)-I\left(B, \lambda_{1} I_{2 n}\right) & =i\left(\lambda_{1} I_{2 n}\right)-\left(I \left(\lambda I_{2 n},\right.\right. \\
\left.\left.\lambda_{1} I_{2 n}\right)+I\left(B, \lambda I_{2 n}\right)\right) & =i\left(\lambda I_{2 n}\right)-I\left(B, \lambda I_{2 n}\right) ;
\end{aligned}
$$

that is, the number $i\left(\lambda I_{2 n}\right)-I\left(B, \lambda I_{2 n}\right)$ is independent of $\lambda \in \mathbb{R}$ with $\lambda I_{2 n}>B$. Hence: 
Definition 2.7. For any $B \in \mathscr{L}_{2 n}^{\infty}$, we define

$$
i(B)=i\left(\lambda I_{2 n}\right)-I\left(B, \lambda I_{2 n}\right),
$$

where $\lambda \in \mathbb{R}$ satisfies $B<\lambda I_{2 n}$.

This index $i(B)$ is monotonously nondecreasing with respect to $B$. That is:

Proposition 2.8. Assume $B_{1}, B_{2} \in \mathscr{L}_{2 n}^{\infty}$ with $B_{1}<B_{2}$. Then $i\left(B_{1}\right)+v\left(B_{1}\right) \leq i\left(B_{2}\right)$.

Proof. By definition,

$$
\begin{aligned}
i\left(B_{2}\right) & =i\left(\lambda I_{2 n}\right)-I\left(B_{2}, \lambda I_{2 n}\right)=i\left(\lambda I_{2 n}\right)-\left(I\left(B_{1}, \lambda I_{2 n}\right)-I\left(B_{1}, B_{2}\right)\right) \\
& =i\left(\lambda I_{2 n}\right)-I\left(B_{1}, \lambda I_{2 n}\right)+I\left(B_{1}, B_{2}\right)=i\left(B_{1}\right)+I\left(B_{1}, B_{2}\right) \\
& \geq i\left(B_{1}\right)+v\left(B_{1}\right) .
\end{aligned}
$$

We next prove that the relative Morse index defined in Definition 2.4 is finite for any $B_{1}, B_{2}$ with $B_{1}<B_{2}$; we also prove Proposition 2.6. To do this, following [Ekeland 1990], we define a Morse type index $i_{\mu}(B)$ for any $B \in \mathscr{L}_{2 n}^{\infty}$, and prove that $I\left(B_{1}, B_{2}\right)=i_{\mu}\left(B_{2}\right)-i_{\mu}\left(B_{1}\right)$. More precisely, for any $B \in \mathscr{L}_{2 n}^{\infty}$, let $\mu \in \mathbb{R} \backslash \pi \mathbb{Z}$ with $B+\mu I_{2 n} \geq I_{2 n}$; then $v\left(-\mu I_{2 n}\right)=0$. The operator $\Lambda x:=J \dot{x}(t)-\mu x(t)$ is invertible and its inverse $\Lambda^{-1}: L^{2} \rightarrow L^{2}$ is self-adjoint and compact, by Proposition 2.5. Define a quadratic form by setting, for $u \in L^{2}$,

$$
\begin{aligned}
q_{\mu, B}(u, u) & =\frac{1}{2} \int_{0}^{1}\left(\left(\left(\Lambda^{-1} u\right)(t), u(t)\right)+\left(C_{\mu}(t) u(t), u(t)\right)\right) d t \\
\left(\bar{C}_{\mu} u, u\right) & :=\int_{0}^{1}\left(C_{\mu}(t) u(t), u(t)\right) d t
\end{aligned}
$$

where $C_{\mu}(t):=\left(\mu I_{2 n}+B(t)\right)^{-1}$. Then $\left(\bar{C}_{\mu} u, u\right)$ defines a Hilbert space structure on $L^{2} . C_{\mu}^{-1} \Lambda^{-1}$ is a self-adjoint and compact operator under this interior product. By spectral theory there is a basis $\left\{e_{j}\right\}_{j \in \mathbb{N}}$ of $L^{2}$ and a sequence $\lambda_{j} \rightarrow 0$ in $\mathbb{R}$ such that

$$
\left(\bar{C}_{\mu} e_{i}, e_{j}\right)=\delta_{i j}, \quad\left(\Lambda^{-1} e_{j}, u\right)=\left(\bar{C}_{\mu} \lambda_{j} e_{j}, u\right) \text { for all } u \in L^{2}
$$

For any $u \in L^{2}$, expressible as $u=\sum_{j=1}^{\infty} \xi_{j} e_{j}$, we have

$$
q_{(\mu, B)}(u, u)=\frac{1}{2} \int_{0}^{1}\left(\left(\Lambda^{-1} u, u\right)+\left(C_{\mu}(t) u, u\right)\right) d t=\frac{1}{2} \sum_{j=1}^{\infty}\left(1+\lambda_{j}\right) \xi_{j}^{2} .
$$


Define

$$
\begin{aligned}
& E_{\mu}^{-}(B):=\left\{\sum_{j=1}^{\infty} \xi_{j} e_{j} \mid \xi_{j}=0 \text { if } 1+\lambda_{j} \geq 0\right\}, \\
& E_{\mu}^{0}(B):=\left\{\sum_{j=1}^{\infty} \xi_{j} e_{j} \mid \xi_{j}=0 \text { if } 1+\lambda_{j} \neq 0\right\}, \\
& E_{\mu}^{+}(B):=\left\{\sum_{j=1}^{\infty} \xi_{j} e_{j} \mid \xi_{j}=0 \text { if } 1+\lambda_{j} \leq 0\right\} .
\end{aligned}
$$

Obviously, $E_{\mu}^{-}(B), E_{\mu}^{0}(B)$ and $E_{\mu}^{+}(B)$ are $q_{(\mu, B)}$-orthogonal and

$$
E_{\mu}^{-}(B) \oplus E_{\mu}^{0}(B) \oplus E_{\mu}^{+}(B)=L^{2} .
$$

Since $\lambda_{j} \rightarrow 0$ as $j \rightarrow \infty$, the spaces $E_{\mu}^{0}(B)$ and $E_{\mu}^{-}(B)$ are finite-dimensional.

Definition 2.9. For any $B \in \mathscr{L}_{2 n}^{\infty}, \mu \in \mathbb{R}$ with $\mu I_{2 n}+B \geq I_{2 n}$, we define

$$
v_{\mu}(B):=\operatorname{dim} E_{\mu}^{0}(B), i_{\mu}(B):=\operatorname{dim} E_{\mu}^{-}(B) .
$$

We call $v_{\mu}(B)$ and $i_{\mu}(B)$ the $\mu$-nullity and $\mu$-index of $B$ respectively.

Proposition 2.10. For any $B_{1}, B_{2}, B \in \mathscr{L}_{2 n}^{\infty}$ with $B_{1}<B_{2}$, we have

$$
v_{\mu}(B)=v(B), \quad I\left(B_{1}, B_{2}\right)=i_{\mu}\left(B_{2}\right)-i_{\mu}\left(B_{1}\right) .
$$

Proof of the first equality. By the definitions, $E_{\mu}^{-}(B), E_{\mu}^{0}(B)$ and $E_{\mu}^{+}(B)$ are $q_{(\mu, B)^{-}}$ orthogonal and satisfy $L^{2}=E_{\mu}^{-}(B) \oplus E_{\mu}^{0}(B) \oplus E_{\mu}^{+}(B)$. For every $u \in E_{\mu}^{0}(B)$, we have

$$
q_{(\mu, B)}(u, v)=0 \text { for any } v \in L^{2} .
$$

So

$$
\Lambda^{-1} u+C_{\mu}(t) u=0 .
$$

Set $x:=\Lambda^{-1} u$. Applying $C_{\mu}(t)=\left(B(t)+\mu I_{2 n}\right)^{-1}$ to both sides and using the equalities $\Lambda=J \frac{d}{d t}-\mu$ and $u=\Lambda x$, we get

$$
\left(B(t)+\mu I_{2 n}\right) x(t)+J \dot{x}(t)-\mu x(t)=0 .
$$

That is,

$$
B(t) x(t)+J \dot{x}(t)=0 .
$$

Hence, $\operatorname{ker}\left(\Lambda_{1}+\bar{B}\right) \cong E_{\mu}^{0}(B)$ and $\nu(B)=v_{\mu}(B)$.

Proof of the second equality. Step 1. We show that if $X$ is a subspace of $L^{2}$ such that $q_{(\mu, B)}(u, u)<0$ for every $u \in X \backslash\{\theta\}$, then $\operatorname{dim} X \leq i_{\mu}(B)$.

In fact, let $e_{1}, \ldots, e_{k}$ be a basis of $X$, we have the decomposition $e_{i}=e_{i}^{-}+e_{i}^{*}$ with $e_{i}^{-} \in E_{\mu}^{-}(B), e_{i}^{*} \in E_{\mu}^{0}(B) \oplus E_{\mu}^{+}(B)$. Suppose there exist numbers $\alpha_{i} \in \mathbb{R}$, 
not all zero, such that $\sum_{i=1}^{k} \alpha_{i} e_{i}^{-}=\theta$. Write $e:=\sum_{i=1}^{k} \alpha_{i} e_{i}$; then $e \in X \backslash\{\theta\}$ and $q_{(\mu, B)}(e, e)<0$; at the same time, $e=\sum_{i=1}^{k} \alpha_{i} e_{i}^{*} \in E_{\mu}^{0}(B) \oplus E_{\mu}^{+}(B)$, and $q_{(\mu, B)}(e, e) \geq 0$, a contradiction. So $\left\{e_{i}^{-}\right\}_{i=1}^{k}$ is linearly independent and $i_{\mu}(B) \geq$ $k=\operatorname{dim} X$.

Step 2. For $B_{1}<B_{2} \in \mathscr{L}_{2 n}^{\infty}$, set $i(\lambda):=i_{\mu}\left((1-\lambda) B_{1}+\lambda B_{2}\right)$ for $\lambda \in[0,1]$. Then $i\left(\lambda_{2}\right) \geq i\left(\lambda_{1}\right)+v\left(\lambda_{1}\right)$ for any $\lambda_{1}, \lambda_{2} \in[0,1]$ with $\lambda_{1}<\lambda_{2}$.

In fact, write $A_{i}=\left(1-\lambda_{i}\right) B_{1}+\lambda_{i} B_{2}$ for $i=1,2$; we only need to prove that

$$
q_{\left(\mu, A_{2}\right)}(u, u)<0 \quad \text { for all } u \in E_{\mu}^{-}\left(A_{1}\right) \oplus E_{\mu}^{0}\left(A_{1}\right) \backslash\{\theta\} .
$$

Take any $u=u^{0}+u^{-}$with $u^{0} \in E_{\mu}^{0}\left(A_{1}\right), u^{-} \in E_{\mu}^{-}\left(A_{1}\right)$. If $u^{-} \neq \theta$ we have

$$
\begin{aligned}
q_{\left(\mu, A_{2}\right)}(u, u) \leq q_{\left(\mu, A_{1}\right)}(u, u) & =q_{\left(\mu, A_{1}\right)}\left(u^{-}, u^{-}\right)+q_{\left(\mu, A_{1}\right)}\left(u^{0}, u^{0}\right) \\
& =q_{\left(\mu, A_{1}\right)}\left(u^{-}, u^{-}\right)<0 .
\end{aligned}
$$

If $u^{-}=\theta$, write $x^{0}=\Lambda^{-1} u^{0}$; then $u^{0}=\Lambda x^{0}$ and $x^{0}$ is a nontrivial solution of

$$
J \dot{x}(t)+A_{1}(t) x(t)=0, \quad P x(0)=\operatorname{Px}(1)=0 .
$$

So $x^{0}(t) \neq 0$ for every $t \in[0,1]$, and $u^{0}(t)=-\left(A_{1}(t)+\mu I_{2 n}\right) x^{0}(t) \neq 0$ for a.e. $t \in(0,1)$. Hence

$$
\begin{aligned}
& \frac{1}{\lambda_{1}-\lambda_{2}} q_{\left(\mu, A_{2}\right)}(u, u) \\
& \quad=\frac{1}{\lambda_{1}-\lambda_{2}}\left(q_{\left(\mu, A_{2}\right)}\left(u^{0}, u^{0}\right)-q_{\left(\mu, A_{1}\right)}\left(u^{0}, u^{0}\right)\right) \\
& =\int_{0}^{1}\left(\left(\mu I_{2 n}+A_{2}(t)\right)^{-1}\left(B_{1}(t)-B_{2}(t)\right)\left(\mu I_{2 n}+A_{1}(t)\right)^{-1} u^{0}(t), u^{0}(t)\right) d t \\
& =\int_{0}^{1}\left(\left(B_{1}(t)-B_{2}(t)\right) x^{0}(t),\left(\mu I_{2 n}+A_{2}(t)\right)^{-1}\left(\mu I_{2 n}+A_{1}(t)\right) x^{0}(t)\right) d t .
\end{aligned}
$$

If $\lambda_{1}=\lambda_{2}$, we have $A_{2}(t)=A_{1}(t)$, and the last integral is

$$
\int_{0}^{1}\left(\left(B_{2}(t)-B_{1}(t)\right) x^{0}(t), x^{0}(t)\right) d t>0 .
$$

Hence, if $\lambda_{2}$ is close to $\lambda_{1}$ and $\lambda_{2}>\lambda_{1}$, we have $q_{\left(\mu, A_{2}\right)}(u, u)<0$. So for $\lambda_{2}<\lambda_{1}$ and $\lambda_{2}$ close to $\lambda_{1}$, we have $i\left(\lambda_{2}\right) \geq i\left(\lambda_{1}\right)+v\left(\lambda_{1}\right)$.

Step 3. For any $\lambda \in[0,1)$, we have $i(\lambda+0)=i(\lambda)+v(\lambda)$.

In fact, from Step $2, i(\lambda)+v(\lambda) \leq i(\lambda+0)$. So we only need to show that $i(\lambda)+v(\lambda) \geq i(\lambda+0)$. Write $k:=i(\lambda+0)$. There exists $\lambda^{\prime}>\lambda$ such that $i(s)=k$ 
and $v(s)=0$ for $s \in\left(\lambda, \lambda^{\prime}\right)$. Set $C(s):=\left(\mu I_{2 n}+(1-s) B_{1}(t)+s B_{2}(t)\right)^{-1}$. By (2-8) we have

$$
\left(C(s) e_{j}^{s}, e_{i}^{s}\right)=\delta_{i j} \quad \text { and } \quad \Lambda^{-1} e_{j}^{s}=C(s) \lambda_{j}^{s} e_{j}^{s} \quad \text { for all } u \in L^{2} .
$$

Since $C(s) \geq\left(\mu I_{2 n}+B_{2}\right)^{-1}$ for $s \in[0,1]$, the sequence $\left\{e_{j}^{s}\right\}$ is bounded in $L^{2}$, and $\lambda_{j}^{s}=\left(\Lambda^{-1} e_{j}^{s}, e_{j}^{s}\right)$ is bounded in $\mathbb{R}$ for $j=1, \ldots, k$ and $s \in[0,1]$. So there exist $s_{l} \in\left(\lambda, \lambda^{\prime}\right)$ such that $s_{l} \rightarrow \lambda+0, e_{j}^{s_{l}} \rightarrow e_{j}$ in $L^{2}, \lambda_{j}^{s_{l}} \rightarrow \lambda_{j}$ in $\mathbb{R}$, and $\Lambda^{-1} e_{j}^{s_{l}} \rightarrow \Lambda^{-1} e_{j}$ in $L^{2}$. Taking the limit in (2-9) we obtain $\left(C(\lambda) e_{j}, e_{i}\right)=\delta_{i j}$ and $\Lambda^{-1} e_{j}=C(\lambda) \lambda_{j} e_{j}$ for $j=1, \ldots, k$. Again for $j=1, \ldots, k$, since $i(s)=k$ for every $s \in\left(\lambda, \lambda^{\prime}\right)$, we have by the definitions $1+\lambda_{j}^{s_{l}}<0$ and $\left\{1 / \lambda_{j}^{s_{l}}\right\}$ bounded in $\mathbb{R}$. So

$$
e_{j}^{s_{l}}=\frac{1}{\lambda_{j}^{s_{l}}} C\left(s_{l}\right)^{-1} \Lambda^{-1} e_{j}^{s_{l}} \rightarrow \frac{1}{\lambda_{j}} C(\lambda)^{-1} \Lambda^{-1} e_{j}=e_{j}
$$

in $L^{2}$. It follows that $\left\{e_{i}\right\}_{i=1}^{k}$ is linearly independent and for every $u=\sum_{j=1}^{k} \alpha_{j} e_{j}$, since $\sum_{j=1}^{k} \alpha_{j} e_{j}^{s_{l}} \rightarrow u$ in $L^{2}$ and

$$
q_{\left(\mu,\left(1-s_{l}\right) B_{1}+s_{l} B_{2}\right)}\left(\sum_{j=1}^{k} \alpha_{j} e_{j}^{s_{l}}, \sum_{j=1}^{k} \alpha_{j} e_{j}^{s_{l}}\right)<0,
$$

taking the limit as $s_{l} \rightarrow \lambda+0$ we have $q_{\left(\mu,(1-\lambda) B_{1}+\lambda B_{2}\right)}(u, u) \leq 0$. In a way similar to the proof of Step 1, this implies $i(\lambda)+v(\lambda) \geq k:=i(\lambda+0)$.

Step 4. The function $i(\lambda)$ is left continuous for $\lambda \in(0,1]$ and continuous for $\lambda \in(0,1)$ with $v(\lambda)=0$.

In fact, from Steps 2 and 3 we only need to show $i(\lambda) \leq i(\lambda-0)$. Let $e_{1}, \ldots, e_{k}$ be a basis of $E^{-}(\lambda):=E_{\mu}^{-}\left((1-\lambda) B_{1}+\lambda B_{2}\right)$, and set

$$
S_{1}:=\left\{\left(\alpha_{1}, \ldots, \alpha_{k}\right) \in \mathbb{R}^{k} \mid \sum_{i=1}^{k} \alpha_{i}^{2}=1\right\} .
$$

Then

$$
\begin{aligned}
& f\left(s, \alpha_{1}, \ldots, \alpha_{k}\right) \\
& \quad:=q_{\left(\mu,(1-s) B_{1}+s B_{2}\right)}\left(\sum_{i=1}^{k} \alpha_{i} e_{i}, \sum_{i=1}^{k} \alpha_{i} e_{i}\right) \\
& =\frac{1}{2} \int_{0}^{1}\left(\left(\Lambda^{-1} \sum_{i=1}^{k} \alpha_{i} e_{i}(t), \sum_{i=1}^{k} \alpha_{i} e_{i}(t)\right)\right. \\
& \left.\quad+\left(\mu I_{2 n}+(1-s) B_{1}(t)+s B_{2}(t)\right)^{-1} \sum_{i=1}^{k} \alpha_{i} e_{i}(t), \sum_{i=1}^{k} \alpha_{i} e_{i}(t)\right) d t
\end{aligned}
$$


is continuous on $[0,1] \times S_{1}$. Since $f\left(\lambda, \alpha_{1}, \ldots, \alpha_{k}\right)<0$ for $\left(\alpha_{1}, \cdots, \alpha_{k}\right) \in S_{1}$ we have $f\left(s, \alpha_{1}, \ldots, \alpha_{k}\right)<0$ for $\left(\alpha_{1}, \ldots, \alpha_{k}\right) \in S_{1}$ and $s$ close enough to $\lambda$. From Step 1 , we have $i(\lambda) \leq i(s)$ for $s$ close to $\lambda$. Hence $i(\lambda) \leq i(\lambda-0)$. In conclusion:

$$
\begin{aligned}
i_{\mu}\left(B_{2}\right) & =i_{\mu}\left(B_{1}\right)+\sum_{0 \leq \lambda<1} v_{\mu}\left((1-\lambda) B_{1}+\lambda B_{2}\right) \\
& =i_{\mu}\left(B_{1}\right)+\sum_{0 \leq \lambda<1} v\left((1-\lambda) B_{1}+\lambda B_{2}\right)=i_{\mu}\left(B_{1}\right)+I\left(B_{1}, B_{2}\right) .
\end{aligned}
$$

Remark. The method of the proof comes from [Ekeland 1990, Theorem I.4.6], with some modifications.

Proof of Proposition 2.6. From Proposition 2.10, fix $\mu \in \mathbb{R}$ with $\mu I_{2 n}+B_{1} \geq I_{2 n}$. Then

$$
\begin{aligned}
I\left(B_{1}, B_{2}\right)+I\left(B_{2}, B_{3}\right) & =i_{\mu}\left(B_{2}\right)-i_{\mu}\left(B_{1}\right)+i_{\mu}\left(B_{3}\right)-i_{\mu}\left(B_{2}\right) \\
& =i_{\mu}\left(B_{3}\right)-i_{\mu}\left(B_{1}\right)=I\left(B_{1}, B_{3}\right) .
\end{aligned}
$$

Proposition 2.11. For any $A \in \mathscr{L}_{n}^{\infty}$, we have

$$
i\left(\operatorname{diag}\left\{A, I_{n}\right\}\right)=m^{-}(A) .
$$

Proof. Fix $\epsilon>0$ and $c>0$ with $c^{2} I_{n}>(1+\epsilon) A$. Since $0 \leq \lambda_{1}<\lambda_{2}<1$, we have

$$
\begin{aligned}
\left(\left(1-\lambda_{2}\right) A+\lambda_{2} c^{2} I_{n}\right)\left(1+\epsilon \lambda_{2}\right)^{-1}-\left(\left(1-\lambda_{1}\right) A+\lambda_{1} c^{2} I_{n}\right)\left(1+\epsilon \lambda_{1}\right)^{-1} & \\
& =\left(\lambda_{2}-\lambda_{1}\right)\left(1+\epsilon \lambda_{1}\right)^{-1}\left(1+\epsilon \lambda_{2}\right)^{-1}\left(c^{2} I_{n}-(1+\epsilon) A\right)>0
\end{aligned}
$$

and

$$
\begin{aligned}
\operatorname{diag}\left\{\left(\lambda_{1} c^{2} I_{n}+\left(1-\lambda_{1}\right) A\right)(1\right. & \left.\left.+\epsilon \lambda_{1}\right)^{-1},\left(1+\epsilon \lambda_{1}\right) I_{n}\right\} \\
& <\operatorname{diag}\left\{\left(\lambda_{2} c^{2} I_{n}+\left(1-\lambda_{2}\right) A\right)\left(1+\epsilon \lambda_{2}\right)^{-1},\left(1+\epsilon \lambda_{2}\right) I_{n}\right\} .
\end{aligned}
$$

As in the proof of Proposition 2.10, we obtain

$$
\begin{aligned}
\sum_{0 \leq \lambda<1} v \operatorname{diag}\left\{\left(\lambda c^{2} I_{n}\right.\right. & \left.+(1-\lambda) A)(1+\epsilon \lambda)^{-1},(1+\epsilon \lambda) I_{n}\right\} \\
& =i_{\mu} \operatorname{diag}\left\{c^{2} I_{n}(1+\epsilon)^{-1},(1+\epsilon) I_{n}\right\}-i_{\mu} \operatorname{diag}\left\{A, I_{n}\right\}
\end{aligned}
$$

where we have dropped the parentheses enclosing the argument of $i_{\mu}$ to lighten the notation. By Proposition 2.10 itself, we have

$$
\begin{aligned}
i_{\mu} \operatorname{diag}\left\{c^{2} I_{n}(1+\epsilon)^{-1},(1+\epsilon)\right. & \left.I_{n}\right\}-i_{\mu} \operatorname{diag}\left\{A, I_{n}\right\} \\
= & I\left(\operatorname{diag}\left\{A, I_{n}\right\}, \operatorname{diag}\left\{\frac{c^{2}}{1+\epsilon} I_{n},(1+\epsilon) I_{n}\right\}\right) .
\end{aligned}
$$

For any $A \in \mathscr{L}_{n}^{\infty}$, consider the system

$$
x^{\prime \prime}+A(t) x=0, \quad x(0)=0=x(1) .
$$


Via the change $z_{1}=x, z_{2}=-(1+\epsilon)^{-1} \dot{z}_{1}$, this system is equivalent to

$$
\dot{z}=J B(t) z, \quad P z(0)=P z(1)=0,
$$

where $B:=\operatorname{diag}\left\{A(1+\epsilon)^{-1},(1+\epsilon) I_{n}\right\}$. So

$$
v\left(\operatorname{diag}\left\{A(1+\epsilon)^{-1},(1+\epsilon) I_{n}\right\}\right)=m^{0}(A) .
$$

It follows that

$$
\sum_{0 \leq \lambda<1} v \operatorname{diag}\left\{\left(\lambda c^{2} I_{n}+(1-\lambda) A\right)(1+\epsilon \lambda)^{-1},(1+\epsilon \lambda) I_{n}\right\}=\sum_{0 \leq \lambda<1} m^{0}\left(\lambda c^{2} I_{n}+(1-\lambda) A\right) .
$$

Denote the right-hand side by $I_{1}\left(A, c^{2} I_{n}\right)$. Again as in Proposition 2.10, we can show that $I_{1}(A, B)=m^{-}(B)-m^{-}(A)$ for any $A, B \in \mathscr{L}_{n}^{\infty}$ with $A<B$. Combining (2-10) and (2-11) with part (1) of Proposition 2.13 below we obtain

$$
i \operatorname{diag}\left\{\frac{c^{2}}{1+\epsilon} I_{n},(1+\epsilon) I_{n}\right\}-i \operatorname{diag}\left\{A, I_{n}\right\}=m^{-}\left(c^{2} I_{n}\right)-m^{-}(A) .
$$

From Definitions 2.4 and 2.7 and equation (2-12), for any $c_{1} \in \mathbb{R}$ with $c_{1}>1+\epsilon$ and $c_{1}>c^{2} /(1+\epsilon)$ we have

$$
\begin{aligned}
i \operatorname{diag}\left\{\frac{c^{2}}{1+\epsilon} I_{n},(1+\epsilon) I_{n}\right\} \\
\quad=i\left(c_{1} I_{2 n}\right)-I\left(\operatorname{diag}\left\{\frac{c^{2}}{1+\epsilon} I_{n},(1+\epsilon) I_{n}\right\}, c_{1} I_{2 n}\right) \\
\quad=i\left(c_{1} I_{2 n}\right)-\sum_{0 \leq \lambda<1} v \operatorname{diag}\left\{\left(\lambda c_{1}+(1-\lambda) \frac{c^{2}}{1+\epsilon}\right) I_{n},\left((1+\epsilon)(1-\lambda)+c_{1} \lambda\right) I_{n}\right\} \\
\quad=i\left(c_{1} I_{2 n}\right)-\sum_{0 \leq \lambda<1} m^{0}\left(\left((1-\lambda) \frac{c^{2}}{1+\epsilon}+\lambda c_{1}\right)\left((1+\epsilon)(1-\lambda)+c_{1} \lambda\right) I_{n}\right) \\
\quad=i\left(c_{1} I_{2 n}\right)-n \operatorname{card}\left\{j \in \mathbb{N} \mid j \in\left[c / \pi, c_{1} / \pi\right)\right\} \\
\quad=i\left(c_{1} I_{2 n}\right)-I\left(c I_{2 n}, c_{1} I_{2 n}\right)=i\left(c I_{2 n}\right)=m^{-}\left(c^{2} I_{2 n}\right) .
\end{aligned}
$$

By (2-13) this proves the result.

Definition 2.12. For any $B_{1}, B_{2} \in \mathscr{L}_{2 n}^{\infty}$, define

$$
I\left(B_{1}, B_{2}\right)=I\left(B_{1}, \mu I_{2 n}\right)-I\left(B_{2}, \mu I_{2 n}\right)
$$

where $\mu \in \mathbb{R}$ satisfies $\mu I_{2 n}>B_{1}, \mu I_{2 n}>B_{2}$.

From Proposition 2.6, $I\left(B_{1}, B_{2}\right)$ is independent of $\mu$ and coincides with the object of Definition 2.4 when $B_{1}<B_{2}$, so it is well defined. 
Proposition 2.13. The index defined by Definitions 2.3 and 2.7, the relative Morse index defined by Definitions 2.4 and 2.12, and the $\mu$-index defined by Definition 2.9 have the following properties:

(1) For any $B_{1}, B_{2} \in \mathscr{L}_{2 n}^{\infty}$, we have

$$
I\left(B_{1}, B_{2}\right)=i\left(B_{2}\right)-i\left(B_{1}\right) .
$$

(2) For any $B_{1}, B_{2}, B_{3} \in \mathscr{L}_{2 n}^{\infty}$, we have

$$
I\left(B_{1}, B_{2}\right)+I\left(B_{2}, B_{3}\right)=I\left(B_{1}, B_{3}\right) .
$$

(3) For any $B \in \mathscr{L}_{2 n}^{\infty}$, there exists $\epsilon_{0}>0$ such that for any $\epsilon \in\left(0, \epsilon_{0}\right]$ we have

$$
\begin{aligned}
& v\left(B+\epsilon I_{2 n}\right)=0=v\left(B-\epsilon I_{2 n}\right), \\
& i\left(B-\epsilon I_{2 n}\right)=i(B), \\
& i\left(B+\epsilon I_{2 n}\right)=i(B)+v(B) .
\end{aligned}
$$

In particular, if $\nu(B)=0$, we have $i\left(B+\epsilon I_{2 n}\right)=i(B)$ for $\epsilon \in\left(0, \epsilon_{0}\right]$.

(4) $i_{\mu}(B)-i(B)$ is a constant for $B$ satisfying $B+\mu I_{2 n} \geq I_{2 n}$, i.e., $i_{\mu}(B)-i(B)=$ $i_{\mu}\left(B_{1}\right)-i\left(B_{1}\right)$ for any other $B_{1} \in \mathscr{L}_{2 n}^{\infty}$ with $B_{1}+\mu I_{2 n} \geq I_{2 n}$. For any $\mu>1$, we have

$$
i_{\mu}(0)=n E\left[\frac{\mu}{\pi}\right] \quad \text { and } \quad i_{\mu}(B)=n E\left[\frac{\mu}{\pi}\right]+n+i(B)
$$

for any $B \in \mathscr{L}_{2 n}^{\infty}$ with $B+\mu I_{2 n} \geq I_{2 n}$.

Proof. (1) follows directly from Definitions 2.7 and 2.12, and (2) follows from (1). We prove the other two parts.

(3) From part (1), we have $i\left(B+I_{2 n}\right)=i(B)+I\left(B, B+I_{2 n}\right)$. From Definition 2.4 and Proposition 2.10, we see that $I\left(B, B+I_{2 n}\right)=\sum_{0 \leq \lambda<1} v\left(B+\lambda I_{2 n}\right)$ is finite. So there is some $\epsilon_{0}>0$ such that $v\left(B+\epsilon I_{2 n}\right)=0$ for $\epsilon \in\left(0, \epsilon_{0}\right]$, and

$$
i\left(B+\epsilon I_{2 n}\right)=i(B)+\sum_{0 \leq \lambda<1} v\left(B+\lambda \epsilon I_{2 n}\right)=i(B)+v(B) .
$$

(4) By Definition 2.9, $i_{\mu}(0)=\operatorname{dim} E_{\mu}^{-}(0)=\sum_{1+\lambda_{j}<0} \operatorname{dim} \operatorname{ker}\left(\Lambda^{-1}-\bar{C}_{\mu} \lambda_{j}\right)$, where $\bar{C}_{\mu}=\mu^{-1} I_{2 n}$. If $\Lambda^{-1} e_{j}-\bar{C}_{\mu} \lambda_{j} e_{j}=0$, letting $\Lambda^{-1} e_{j}=x_{j}$, we have

$$
J \dot{x}_{j}-\mu\left(1+\frac{1}{\lambda_{j}}\right) x_{j}=0 .
$$

So $k \pi=\mu\left(1+\frac{1}{\lambda_{j}}\right)$ for $k \in \mathbb{Z}$, and $1+\lambda_{j}=\frac{\mu}{k \pi-\mu}+1$. Since

$$
1+\lambda_{j}<0 \Longleftrightarrow\{k \pi-\mu<0, k \pi>0\} \Longleftrightarrow 0<k<\frac{\mu}{\pi},
$$


we have $\operatorname{ker}\left(\Lambda^{-1}-\bar{C}_{\mu} \lambda_{j}\right) \cong \operatorname{ker}\left(\Lambda_{1}+\bar{B}\right)$, where $\bar{B} x=-k \pi x$ and $\Lambda_{1} x=J \dot{x}$. Hence $\operatorname{dim} \operatorname{ker}\left(\Lambda^{-1}-\bar{C}_{\mu} \lambda_{j}\right)=n$, and $i_{\mu}(0)=n E\left[\frac{\mu}{\pi}\right]$. This is the first displayed equality in (4). From (1) and Proposition 2.10, we have

$$
i_{\mu}\left(B_{1}\right)-i_{\mu}(B)=I\left(B, B_{1}\right)=i\left(B_{1}\right)-i(B) .
$$

So

$$
i_{\mu}(B)-i(B)=i_{\mu}(0)-i(0) .
$$

From Definition 2.3, we have $i(0)=-n$, and the second desired equality follows.

\section{Existence and multiplicity of solutions for nonlinear Hamiltonian systems}

Consider the problem

$$
\dot{x}=J H^{\prime}(t, x), \quad P x(0)=0=P x(1),
$$

where $H:[0,1] \times \mathbb{R}^{2 n} \rightarrow \mathbb{R}$ is continuous and $H^{\prime}(t, x)$ is its gradient with respect to $x$. (For the definition of $P$ see immediately after (1-2).) A problem of the form (3-1) is called a Bolza problem by I. Ekeland. We will always assume that $H^{\prime}:[0,1] \times \mathbb{R}^{2 n} \rightarrow \mathbb{R}^{2 n}$ is continuous as well.

Theorem 3.1. Assume that $H$ and $H^{\prime}$ are continuous and that

(1) $H^{\prime}(t, x)=B(t, x) x+o(|x|)$ as $|x| \rightarrow+\infty$, where $B(t, x)$ is a symmetric $2 n \times 2$ mmatrix and continuous with respect to $(t, x) \in[0,1] \times \mathbb{R}^{2 n}$;

(2) there exist $B_{1}(t)$ and $B_{2}(t)$ such that $B_{1}(t) \leq B(t, x) \leq B_{2}(t), i\left(B_{2}\right)=i\left(B_{1}\right)$ and $v\left(B_{2}\right)=0$.

Then (3-1) has at least one solution.

Proof. Consider

$$
J \dot{x}+\lambda B_{1}(t) x+(1-\lambda) H^{\prime}(t, x)=0, \quad P x(0)=0=P x(1),
$$

where $\lambda \in(0,1)$. Set

$C_{0}\left([0,1] ; \mathbb{R}^{2 n}\right):=\left\{x:[0,1] \rightarrow \mathbb{R}^{2 n} \mid \operatorname{Px}(0)=0=\operatorname{Px}(1)\right.$ and $x(t)$ is continuous for $t \in[0,1]\}$,

with the norm $\|x\|_{C}=\max _{t \in[0,1]}|x(t)|$. We only need to show that there exists $r>0$ such that $\left\|x_{\lambda}\right\|_{C}<r$ for any solution $x_{\lambda}$ of (3-2). In fact, let $(\Lambda x)(t)=$ $J \dot{x}(t)-\mu x(t),(\bar{C} x)(t)=\left(\mu I_{2 n}+B_{1}(t)\right) x(t),(N x)(t)=\mu x(t)+H^{\prime}(t, x(t))$. Then $\Lambda^{-1} \bar{C}: C_{0}\left([0,1] ; \mathbb{R}^{2 n}\right) \rightarrow C_{0}\left([0,1] ; \mathbb{R}^{2 n}\right)$ is linear and compact, $\Lambda^{-1} N$ : $C_{0}\left([0,1] ; \mathbb{R}^{2 n}\right) \rightarrow C_{0}\left([0,1] ; \mathbb{R}^{2 n}\right)$ is compact, and $\operatorname{ker}\left(\Lambda^{-1} \bar{C}+\mathrm{id}\right)=\{\theta\}$. We have the Leray-Schauder degree $\operatorname{deg}\left(\mathrm{id}+\Lambda^{-1} \bar{C}, B_{r}, \theta\right) \neq 0$. By homotopy invariance 
we have $\operatorname{deg}\left(\mathrm{id}+\Lambda^{-1} N, B_{r}, \theta\right)=\operatorname{deg}\left(\mathrm{id}+\Lambda^{-1} \bar{C}, B_{r}, \theta\right) \neq 0$, and $\Lambda^{-1} N x+x=0$, so (3-1) has a solution.

Now we prove that the solution $x_{\lambda}$ of (3-2) is bounded. Assume to the contrary that there exists a sequence $\left\{x_{k}\right\}$ in $C\left([0,1] ; \mathbb{R}^{2 n}\right)$ such that $\left\|x_{k}\right\|_{C} \rightarrow+\infty,\left\{\lambda_{k}\right\} \subset$ $(0,1)$, and

$$
\begin{gathered}
J \dot{x}_{k}+\lambda_{k} B_{1}(t) x_{k}(t)+\left(1-\lambda_{k}\right) H^{\prime}\left(t, x_{k}(t)\right)=0, \\
P x_{k}(0)=0=P x_{k}(1) .
\end{gathered}
$$

Set $y_{k}=x_{k} /\left\|x_{k}\right\|_{C}$ and $A_{k}(t)=\lambda_{k} B_{1}(t)+\left(1-\lambda_{k}\right) B\left(t, x_{k}(t)\right)$; also define $e_{k}(t)=$ $\left(1-\lambda_{k}\right)\left(H^{\prime}\left(t, x_{k}(t)\right)-B\left(t, x_{k}(t)\right) x_{k}(t)\right)\left\|x_{k}\right\|_{C}^{-1}$. Then

$$
J \dot{y}_{k}(t)+A_{k}(t) y_{k}(t)+e_{k}(t)=0, \quad P y_{k}(0)=0=P y_{k}(1) .
$$

From assumption (2), $\left\{\dot{y}_{k}\right\}$ is bounded in $C_{0}\left([0,1], \mathbb{R}^{2 n}\right)$. By the Ascoli-Arzelà theorem we may assume that $y_{k} \rightarrow y_{0}$ in $C_{0}\left([0,1] ; \mathbb{R}^{2 n}\right)$. From assumption (1), we have $e_{k} \rightarrow 0$ in $C\left([0,1] ; \mathbb{R}^{2 n}\right)$. From assumption (2) again we have $B_{1} \leq A_{k} \leq B_{2}$. Now write $A_{k}(t)=\left(a_{i j}^{(k)}(t)\right)_{2 n \times 2 n}$ and $B_{k}(t)=\left(b_{i j}^{(k)}(t)\right)_{2 n \times 2 n}$ for $k=1,2, \ldots$ Then $b_{i i}^{(1)} \leq a_{i i}^{(k)} \leq b_{i i}^{(2)} \quad$ and $\quad 2 b_{i j}^{(1)}+b_{i i}^{(1)}+b_{j j}^{(1)} \leq 2 a_{i j}^{(k)}+a_{i i}^{(k)}+a_{j j}^{(k)} \leq 2 b_{i j}^{(2)}+b_{i i}^{(2)}+a_{j j}^{(2)}$

for all distinct $i$ and $j$. We may further assume $\lambda_{k} \rightarrow \lambda_{0}$ in $\mathbb{R}$ and $a_{i j}^{(k)} \rightarrow a_{i j}$ in $L^{2}(0,1)$ by going to subsequences if necessary. Write $A_{0}(t)=\left(a_{i j}(t)\right)_{2 n \times 2 n}$; integrating (3-3) and taking the limit we have

$$
J \dot{y}_{0}+A_{0}(t) y_{0}(t)=0, \quad P y_{0}(0)=0=P y_{0}(1) .
$$

Since $B_{1} \leq A_{k} \leq B_{2}$, it follows that $B_{1} \leq A_{0} \leq B_{2}$, and $A_{0} \in \mathscr{L}_{2 n}^{\infty}$. Because $i\left(B_{2}\right)=i\left(B_{1}\right)$ and $v\left(B_{2}\right)=0$, we see from Proposition 2.8 that $v\left(A_{0}\right)=0$. This contradicts the fact that $y_{0}$ is a nontrivial solution of (3-4).

Theorem 3.2. Assume that $H$ and $H^{\prime}$ are continuous and that there exist $B_{1}, B_{2} \in$ $\mathscr{L}_{2 n}^{\infty}$ satisfying the following properties:

(1) $B_{1} \leq B_{2}$.

(2) $H$ is $\left(B_{1}, B_{2}\right)$-subquadratic at infinity, i.e., the difference

$$
N(t, x):=H(t, x)-\frac{1}{2}\left(B_{1}(t) x, x\right)
$$

is strict convex with respect to $x$ for all $t \in[0,1]$, and there exists $c \in \mathbb{R}$ such that $H(t, x) \leq \frac{1}{2}\left(B_{2}(t) x, x\right)+c$ for all $(t, x)$.

(3) $i\left(B_{2}\right)=i\left(B_{1}\right)+v\left(B_{1}\right)$, and $v\left(B_{2}\right)=0$ if $B_{1}<B_{2}$.

Then (3-1) has one solution. 
Proof. Step 1. Let $\Gamma: W \subset L^{2} \rightarrow L^{2}$ be defined by $(\Gamma x)(t):=J \dot{x}(t)+B_{1}(t) x(t)$. We first prove that the range $R(\Gamma):=\Gamma(W)$ is closed in $L^{2}$, that

$$
L^{2}=\operatorname{ker}(\Gamma) \oplus R(\Gamma),
$$

that the restriction $\Gamma_{0}:=\left.\Gamma\right|_{R(\Gamma)}$ is invertible, and that $\Gamma_{0}^{-1}: R(\Gamma) \rightarrow R(\Gamma)$ is self-adjoint and compact.

In fact, if $v\left(B_{1}\right)=0$, by Proposition 2.5, we have $\operatorname{ker} \Gamma=\{\theta\}$ and $R(\Gamma)=L^{2}$. Thus we need only consider the case $v\left(B_{1}\right) \neq 0$. From Proposition 2.13, there exists $\epsilon>0$ such that $v\left(B_{1}+\epsilon I_{2 n}\right)=0$. From Proposition 2.5(2), there exist $\lambda_{j} \in \mathbb{R}$ with $\lambda_{j} \rightarrow \pm \infty$ as $\lambda_{j} \rightarrow \pm \infty$ such that

$$
L^{2}=\bigoplus_{j=-\infty}^{+\infty} E_{j}, \quad \text { where } E_{j}:=\operatorname{ker}\left(J \frac{d}{d t}+B_{1}(\cdot)+\epsilon I_{2 n}-\lambda_{j} I_{2 n}\right)
$$

Since $v\left(B_{1}\right)=\operatorname{dim} \operatorname{ker}\left(J(d / d t)+B_{1}(\cdot)\right) \neq 0$ and since $\Gamma u=\left(\lambda_{j}-\epsilon\right) u$ for all $u \in E_{j}$, there exists some $j_{0} \in \mathbb{Z}$ such that $\epsilon-\lambda_{j_{0}}=0$. Then $E_{j_{0}}=\operatorname{ker} \Gamma, R(\Gamma)=$ $\bigoplus_{j=-\infty, j \neq j_{0}}^{+\infty} E_{j}$, and $L^{2}=\operatorname{ker}(\Gamma) \oplus R(\Gamma)$. Now, given $u \in R(\Gamma)$, write

$$
u=\sum_{j=-\infty, j \neq j_{0}}^{+\infty} u_{j}, \quad \text { with } \sum_{j=-\infty, j \neq j_{0}}^{+\infty}\left\|u_{j}\right\|_{L^{2}}^{2}<+\infty .
$$

Since $\Gamma u_{j}=\left(\lambda_{j}-\epsilon\right) u_{j}$, we have $\Gamma_{0}^{-1} u_{j}=\frac{1}{\lambda_{j}-\epsilon} u_{j}$. So

$$
\Gamma_{0}^{-1} u=\sum_{j=-\infty, j \neq j_{0}}^{+\infty} \frac{1}{\lambda_{j}-\epsilon} u_{j}
$$

Since $\lambda_{j} \rightarrow \infty, \Gamma_{0}^{-1}: R(\Gamma) \rightarrow R(\Gamma)$ is compact and self-adjoint.

Step 2. Consider the functional

$$
\psi(u):=\int_{0}^{1}\left(\frac{1}{2}\left(\Gamma_{0}^{-1} u, u\right)+N^{*}(t,-u)\right) d t,
$$

where $N^{*}(t, u)=\sup _{v \in \mathbb{R}^{2 n}}\{(u, v)-N(t, v)\}$ is the Fenchel conjugate of $N(t, u)$. From the continuity of $H$ and $H^{\prime}$ as well as the definition and the strict convexity of $N$, we conclude that the gradient $N^{* \prime}(t, u)$ of $N^{*}(t, u)$ with respect to $u$ exists and is continuous with respect to $u$, so $\psi \in C^{1}\left(L^{2}, \mathbb{R}\right)$ and

$$
\left(\psi^{\prime}(u), v\right)=\int_{0}^{1}\left(\left(\Gamma_{0}^{-1} u, v\right)+\left(N^{* \prime}(t,-u),-v\right)\right) d t
$$

for all $v \in R(\Gamma)$. In view of Proposition 2.13 and of $v\left(B_{2}\right)=0$, there exists $\epsilon>0$ such that $v\left(B_{2}+\epsilon I_{2 n}\right)=0$ and $i\left(B_{2}+\epsilon I_{2 n}\right)=i\left(B_{2}\right)$. So without loss of generality 
we assume that, for some $\epsilon>0$,

$$
B_{2}-B_{1} \geq \epsilon I_{2 n} .
$$

Write $C(t):=\left(B_{2}(t)-B_{1}(t)\right)^{-1}$. Then $(\bar{C} u, v):=\int_{0}^{1}(C(t) u(t), v(t)) d t$ defines a Hilbert inner product structure on $R(\Gamma)$. By the spectral theory of self-adjoint compact operators we find a basis of $R(\Gamma)$, denoted by $\left\{e_{j}\right\}$, and a real sequence $\left\{\lambda_{j}\right\}$ with $\lambda_{j} \rightarrow 0$ satisfying

$$
\left(\bar{C} e_{i}, e_{j}\right)=\delta_{i j} \quad \text { and } \quad\left(\Gamma_{0}^{-1} e_{j}, u\right)=\lambda_{j}\left(\bar{C} e_{j}, u\right) \text { for all } u \in R(\Gamma) .
$$

From (3-5), there exists $\xi_{j} \in \operatorname{ker} \Gamma$ such that

$$
\lambda_{j} C(t) e_{j}-\Gamma_{0}^{-1} e_{j}=\xi_{j} .
$$

Let $x_{j}=\Gamma_{0}^{-1} e_{j}+\xi_{j}$. We have $\Gamma x_{j}-\lambda_{j}^{-1}\left(B_{2}(t)-B_{1}(t)\right) x_{j}=0$. Since $i\left(B_{2}\right)=$ $i\left(B_{1}\right)+v\left(B_{1}\right)$ and $v\left(B_{2}\right)=0$, we have $v\left(B_{1}+\lambda\left(B_{2}-B_{1}\right)\right)=0$ for any $\lambda \in(0,1]$ by Definition 2.4 and Proposition 2.13(1). Hence, for $\lambda_{j}<0$ we have $-\lambda_{j}^{-1}>1$ and $\lambda_{j}+1>0$. So for any $j \in \mathbb{N}$, we have $1+\lambda_{j}>0$. Set $\bar{\lambda}:=\inf \left\{\lambda_{j}<0 \mid j \in \mathbb{N}\right\}$. Since $\lambda_{j} \rightarrow 0$ as $j \rightarrow \infty$, we have

$$
1+\bar{\lambda}>0 .
$$

For any $u=\sum_{i=1}^{\infty} \xi_{i} e_{i} \in R(\Gamma)$, we get from (3-9)

$$
\left(\Gamma_{0}^{-1} u, u\right)=\sum_{j=1}^{\infty} \xi_{j}\left(\Gamma_{0}^{-1} u, e_{j}\right)=\sum_{i=1}^{\infty} \lambda_{j}\left|\xi_{j}\right|^{2} \geq \bar{\lambda}(\bar{C} u, u) .
$$

On the other hand, we know that $N(t, u) \leq \frac{1}{2}\left(\left(B_{2}(t)-B_{1}(t)\right) u, u\right)+c$ for all $(t, u)$, by assumption (2) of the theorem. Thus

$$
N^{*}(t, u) \geq \frac{1}{2}(C(t) u, u)-c
$$

and

$$
\int_{0}^{1} N^{*}(t,-u) d t \geq \frac{1}{2}(\bar{C} u, u)-c
$$

for all $u \in R(\Gamma)$. Combining this with (3-10), (3-11) and (3-6), we obtain

$$
\psi(u) \geq(\bar{\lambda}+1)(\bar{C} u, u)-c \rightarrow+\infty
$$

as $(\bar{C} u, u) \rightarrow+\infty$. Let $\left\{u_{j}\right\} \subset R(\Gamma)$ satisfy $\psi\left(u_{n}\right) \rightarrow \inf _{u \in R(\Gamma)} \psi(u)$. Then $\left\{u_{j}\right\}$ is bounded in $R(\Gamma)$, and there exists a subsequence, denoted again by $\left\{u_{j}\right\}$, converging weakly to $u_{0}$ in $R(\Gamma)$. As in [Ekeland 1990, Theorem II.2.1], $\psi$ is weakly lower semicontinuous, so $\inf _{u \in R(\Gamma)} \psi(u)=\lim \psi\left(u_{j}\right) \geq \psi\left(u_{0}\right)$, and $\psi\left(u_{0}\right)=\inf _{u \in R(\Gamma)} \psi(u)$. Therefore, $\psi^{\prime}\left(u_{0}\right)=0$. 
By (3-7), we have

$$
\int_{0}^{1}\left(\Gamma_{0}^{-1} u_{0}-N^{* \prime}\left(t,-u_{0}\right), v\right) d t=0
$$

for all $v \in R(\Gamma)$. From (3-5) again, $\xi_{0}:=\Gamma_{0}^{-1} u_{0}-N^{* \prime}\left(t,-u_{0}\right)$ lies in $\operatorname{ker} \Gamma$. Writing $\Gamma_{0}^{-1} u_{0}-\xi_{0}=x_{0}$, we get

$$
J \dot{x}_{0}(t)+B_{1}(t) x_{0}(t)+N^{\prime}\left(t, x_{0}(t)\right)=0, \quad \operatorname{Px}(0)=0=\operatorname{Px}(1) .
$$

The proof is complete.

Remark. As in [Ekeland 1990, Theorem III.2.1], it is not necessary to assume that $N(t, u)$ is strictly convex with respect to $u$ : simple convexity suffices. We used the strict condition only in order to derive that $N^{*}(t, u)$ is differentiable with respect to $u$, to simplify the proof.

Corollary 3.3. Assume $B_{1}, B_{2} \in \mathscr{L}_{2 n}^{\infty}, B_{1}(t), B_{2}(t)$ are continuous with respect to $t$, and satisfy assumption (1) of Theorem 3.2. Let $\gamma_{i}(t)$ with $0 \leq \gamma_{1}(t) \leq \cdots \leq \gamma_{2 n}(t)$ be the eigenvalues of $B_{2}(t)-B_{1}(t)$, and let $\lambda_{j}$ be the eigenvalues of $\Gamma$, and set:

$$
\gamma:=\max \left\{\gamma_{2 n}(t) \mid 0 \leq t \leq 1\right\}, \quad \lambda:=\max \left\{\lambda_{j}<0\right\} .
$$

Then (3-1) has a solution if $|\lambda|>\gamma$.

Proof. We only need to prove that $v\left(B_{2}\right)=0$ and $i\left(B_{2}\right)=i\left(B_{1}\right)+v\left(B_{1}\right)$. In fact, by the definition of $\gamma$ we have $B_{2}(t)-B_{1}(t) \leq \gamma I_{2 n}$, and $B_{2}(t) \leq B_{1}(t)+\gamma I_{2 n}$. Since $-\lambda=|\lambda|>\gamma$, then $\Gamma x+\mu x=0$ has no nontrivial solutions for any $\mu \in(0, \gamma]$. By Proposition 2.13 and Definition 2.4, we have $i\left(B_{1}+\gamma I_{2 n}\right)=i\left(B_{1}\right)+v\left(B_{1}\right)$. Since $B_{1}<B_{2} \leq B_{1}+\gamma I_{2 n}$, we have $i\left(B_{1}\right)+v\left(B_{1}\right) \leq i\left(B_{2}\right), i\left(B_{2}\right)+v\left(B_{2}\right) \leq i\left(B_{1}+\gamma I_{2 n}\right)$. So $v\left(B_{2}\right)=0$ and $i\left(B_{2}\right)=i\left(B_{1}\right)+v\left(B_{1}\right)$.

Corollary 3.4. Assume that

$$
((k-1) \pi+\epsilon) I_{2 n} \leq H^{\prime \prime}(t, x) \leq(k \pi-\epsilon) I_{2 n} \quad \text { for all }(t, x),
$$

where $\epsilon>0$ is small and $k \in \mathbb{Z}$. Then (3-1) has at least one solution.

Proof. Take $B_{1}(t):=(k-1) \pi I_{2 n}$ and $B_{2}(t):=\left(k \pi-\frac{1}{2} \epsilon\right) I_{2 n}$. Then $N(t, x):=$ $H(t, x)-\frac{1}{2}\left(B_{1}(t) x, x\right)$ is convex with respect to $x$, since $N^{\prime \prime}(t, x) \geq \epsilon I_{2 n}$, and

$$
\begin{aligned}
H(t, x) & =\int_{0}^{1} d s\left(\int_{0}^{1} H^{\prime \prime}(t, \tau s x) x s d \tau, x\right)+\left(H^{\prime}(t, \theta), x\right)+H(t, \theta) \\
& \leq \frac{1}{2}(k \pi-\epsilon)|x|^{2}+\left(H^{\prime}(t, \theta), x\right)+H(t, \theta) \\
& \leq \frac{1}{2} b|x|^{2}+c,
\end{aligned}
$$

where $b:=k \pi-\frac{1}{2} \epsilon$ and $c>0$ is a constant. Since $i\left(b I_{2 n}\right)=i\left((k-1) \pi I_{2 n}\right)+$ $v\left((k-1) \pi I_{2 n}\right)$, the hypotheses of Theorem 3.2 are satisfied. 
Example 3.5. Let

$$
b(t)=\left\{\begin{array}{cl}
\frac{\pi}{2\left(1-t_{1}\right)} & \text { for } t \in\left(t_{1}, 1\right), \\
\frac{\pi}{2 t_{1}} & \text { for } t \in\left(0, t_{1}\right) .
\end{array}\right.
$$

Then $i\left(b I_{2 n}\right)=\sum_{\lambda \in[0,1)} v\left(\lambda b I_{2 n}\right)-n=0$. Let $H(t, x)=(b(t)-\epsilon)|x|^{2}+|x|^{\epsilon+1}+$ $h(t)$ and $\epsilon \in(0,1)$ is fixed. By Theorem 3.2, (3-1) has a solution. But $\gamma_{i}(t)=$ $b(t)-\epsilon \rightarrow+\infty$ as $t_{1} \rightarrow 0^{+}$for $t \in\left(0, t_{1}\right)$. So the assumptions in Corollary 3.3 are not satisfied.

Remark. Corollary 3.3 can be compared with [Ekeland 1990, Theorem II.2.1]. There Ekeland discusses periodic solutions of the Hamiltonian system consisting of the first equation in (3-1), and proves existence under similar conditions.

We now discuss the multiplicity for solutions of (3-1).

Theorem 3.6. Let the assumptions of Theorem 3.1 be satisfied, and assume moreover that

$$
H^{\prime}(t, x)=A(t, x) x+o(|x|) \quad \text { for small }|x|,
$$

where $A(t, x)$ is a symmetric $2 n \times 2 n$ matrix varying continuously with $(t, x) \in$ $[0,1] \times \mathbb{R}^{2 n}$ and satisfying $A_{1}(t) \leq A(t, x) \leq A_{2}(t)$, for all $(t, x)$, where $A_{1}$ and $A_{2}$ are such that $i\left(A_{1}\right)=i\left(A_{2}\right)$ and $v\left(A_{2}\right)=0$.

Then (3-1) has a nontrivial solution if $i\left(B_{1}\right)-i\left(A_{1}\right)$ is odd.

We will use Leray-Schauder degree theory to prove the theorem.

Lemma 3.7 [Chang 1986, Chapter 1, Proposition 4.1']. Assume that $K: X \rightarrow X$ is a linear compact operator and $\sigma(K)$, the spectral set of $K$, does not contain -1 . Then the Leray-Schauder degree $\operatorname{deg}(\mathrm{id}+K, \Omega, \theta)$ is $(-1)^{\beta}$, where $\beta=\sum_{\lambda_{j}+1<0, \lambda_{j} \in \sigma(K)} \beta_{j}$ and $\beta_{j}=\operatorname{dim} \bigcup_{k=1}^{\infty} \operatorname{ker}\left(K-\lambda_{j} \text { id }\right)^{k}$.

Proof of Theorem 3.6. We use the notations in Theorem 3.1. For $\mu \in \mathbb{R}$ with $\mu I_{2 n}+B_{1} \geq I_{2 n}$ and $\mu I_{2 n}+A_{1} \geq I_{2 n}$, we want to show that there exist $r_{1}, r \in \mathbb{R}$ with $r>r_{1}>0$ such that

$$
\begin{aligned}
& \operatorname{deg}\left(\mathrm{id}+\Lambda^{-1} N, B_{r}, \theta\right)=(-1)^{i_{\mu}\left(B_{1}\right)}, \\
& \operatorname{deg}\left(\mathrm{id}+\Lambda^{-1} N, B_{r_{1}}, \theta\right)=(-1)^{i_{\mu}\left(A_{1}\right)} .
\end{aligned}
$$

From Proposition 2.13, we have $i_{\mu}\left(B_{1}\right)-i_{\mu}\left(A_{1}\right)=i\left(B_{1}\right)-i\left(A_{1}\right)$, and hence $\operatorname{deg}\left(\mathrm{id}+\Lambda^{-1} N, B_{r} \backslash B_{r_{1}}, \theta\right)=(-1)^{i_{\mu}\left(B_{1}\right)}-(-1)^{i_{\mu}\left(A_{1}\right)} \neq 0$, so the problem (3-1) has a nontrivial solution $x=x(t)$ with $r>\|x\|_{C}>r_{1}$. We only prove (3-12) since in a similar way we can get (3-13). From the proof of Theorem 3.1, we have

$$
\operatorname{deg}\left(\mathrm{id}+\Lambda^{-1} N, B_{r}, \theta\right)=\operatorname{deg}\left(\mathrm{id}+\Lambda^{-1} \bar{C}, B_{r}, \theta\right) .
$$


Set $K=\Lambda^{-1} \bar{C}$. If $\lambda \in \sigma(K)$ with $1+\lambda<0$, we have $K x=\lambda x$ for some $x \neq \theta$. Set $y(t):=\left(\mu I_{2 n}+B_{1}(t)\right) x(t)$; then $\Lambda^{-1} y=\bar{C}^{-1} \lambda y$, and $\operatorname{ker}(K-\lambda$ id $) \cong \operatorname{ker}\left(\Lambda^{-1}-\right.$ $\left.\bar{C}^{-1} \lambda\right)$. From the definitions,

$$
\begin{aligned}
E_{\mu}^{-}\left(B_{1}\right) & =\bigoplus_{\lambda+1<0} \operatorname{ker}\left(\Lambda^{-1}-\bar{C}^{-1} \lambda\right) \cong \bigoplus_{\lambda+1<0} \operatorname{ker}(K-\lambda \mathrm{id}), \\
i_{\mu}\left(B_{1}\right) & =\operatorname{dim} E_{\mu}^{-}\left(B_{1}\right)=\operatorname{dim} \bigoplus_{\lambda+1<0} \operatorname{ker}(K-\lambda \mathrm{id}) .
\end{aligned}
$$

By Lemma 3.7, in order to prove (3-12) we only need to prove that

$$
\operatorname{ker}(K-\lambda \mathrm{id})^{2}=\operatorname{ker}(K-\lambda \mathrm{id}) .
$$

From (2-8), we have an orthogonal decomposition

$$
L^{2}=R\left(\Lambda^{-1}-\bar{C}^{-1} \lambda\right) \oplus \operatorname{ker}\left(\Lambda^{-1}-\bar{C}^{-1} \lambda\right) .
$$

If $(K-\lambda \text { id })^{2} x=\theta$, let $\bar{x}:=(K-\lambda$ id $) x=\Lambda^{-1} \bar{C} x-\lambda x$. Then

$$
\bar{x}=\left(\Lambda^{-1}-\lambda \bar{C}^{-1}\right)(\bar{C} x) .
$$

Since $(K-\lambda$ id $) \bar{x}=0$, we have $\bar{C} \bar{x} \in \operatorname{ker}\left(\Lambda^{-1}-\lambda \bar{C}^{-1}\right)$. From (3-15) we get $(\bar{C} \bar{x}, \bar{x})=0$ and $\bar{x}=\theta$. This proves $(3-14)$.

To conclude we will use Morse theory to discuss the multiplicity of solutions. We will make the assumption that the second derivative $H^{\prime \prime}:[0,1] \times \mathbb{R}^{2 n} \rightarrow \mathbb{R}^{2 n \times 2 n}$ is continuous.

Theorem 3.8. Assume that $H, H^{\prime}, H^{\prime \prime}$ are all continuous, that $H(t, \theta) \equiv 0$, that $H^{\prime}(t, \theta) \equiv \theta$ and that the following conditions are satisfied:

(1) There exist $B_{1}, B_{2} \in \mathscr{L}_{2 n}^{\infty}$ with $i\left(B_{2}\right)=i\left(B_{1}\right), v\left(B_{2}\right)=0$ such that

$$
B_{1}(t) \leq H^{\prime \prime}(t, x) \leq B_{2}(t) \quad \text { for all }(t, x) \text { with }|x| \geq r>0 .
$$

(2) With $B_{0}(t):=H^{\prime \prime}(t, \theta)$, we have

$$
i\left(B_{1}\right) \notin\left[i\left(B_{0}\right), i\left(B_{0}\right)+v\left(B_{0}\right)\right] .
$$

Then (3-1) has one nontrivial solution. Moreover, under the further assumption that

(3) $0=v\left(B_{0}\right)$ and $\left|i\left(B_{1}\right)-i\left(B_{0}\right)\right| \geq n$, equation (3-1) has two nontrivial solutions.

Proof. From assumption (1), $H^{\prime \prime}(t, x)$ is bounded and there exist $\mu_{1}, \mu>0$ such that

$$
\mu_{1} I_{2 n} \geq H^{\prime \prime}(t, x)+\mu I_{2 n} \geq I_{2 n} \text { for all }(t, x)
$$


Recall that $(\Lambda x)(t)=J \dot{x}(t)-\mu x(t)$. Define $N(t, x)=H(t, x)+\frac{1}{2} \mu|x|^{2}$ and $N^{*}(t, x)=\sup _{y \in \mathbb{R}^{2 n}}\{(x, y)-N(t, y)\}$. From [Ekeland 1990, Proposition II.2.10] we have

$$
N^{* \prime \prime}\left(t, u^{*}\right)=N^{\prime \prime}(t, u)^{-1}
$$

if $u=N^{* \prime}\left(t, u^{*}\right)$, or equivalently if $u^{*}=N^{\prime}(t, u)$ (by the Fenchel conjugate formula; see [Ekeland 1990, Proposition II.1.15]). By (3-16) we have

$$
I_{2 n} \leq N^{* \prime \prime}\left(t, u^{*}\right) \leq \mu_{1}^{-1} I_{2 n} \quad \text { for all }(t, u) \in[0,1] \times \mathbb{R}^{2 n},
$$

and hence $|u| \rightarrow+\infty$ if and only if $\left|u^{*}\right| \rightarrow+\infty$. From assumption (1) and (3-17) there exists $r_{1}>0$ such that

$$
\left(B_{2}(t)+\mu I_{2 n}\right)^{-1} \leq N^{* \prime \prime}\left(t, u^{*}\right) \leq\left(B_{1}(t)+\mu I_{2 n}\right)^{-1}
$$

for all $\left(t, u^{*}\right) \in[0,1] \times \mathbb{R}^{2 n}$ such that $\left|u^{*}\right| \geq r_{1}$. Consider the functional defined by

$$
\psi(u)=\int_{0}^{1}\left(\frac{1}{2}\left(\Lambda^{-1} u(t), u(t)\right)+N^{*}(t, u(t))\right) d t \quad \text { for all } u \in L^{2} .
$$

We prove that $\psi$ satisfies the Palais-Smale condition. Assume that $\left\{u_{j}\right\}$ is a sequence in $L^{2}$ such that $\psi\left(u_{j}\right)$ is bounded and $\psi^{\prime}\left(u_{j}\right) \rightarrow \theta$ in $L^{2}$. From $N^{\prime}(t, \theta) \equiv \theta$, we have $N^{* \prime}(t, \theta) \equiv \theta$ and

$$
\left(\psi^{\prime}(u), v\right)=\int_{0}^{1}\left(\left(\Lambda^{-1} u(t), v(t)\right)+\left(N^{* \prime}(t, u(t)), v(t)\right)\right) d t
$$

for all $v \in L^{2}$. Noticing that $\int_{0}^{1} N^{* \prime \prime}\left(t, \theta u_{j}(t)\right) d \theta u_{j}(t)=N^{* \prime}\left(t, u_{j}(t)\right)$, we have

$$
\Lambda^{-1} u_{j}+\int_{0}^{1} N^{* \prime \prime}\left(t, \theta u_{j}(t)\right) d \theta u_{j}=\psi^{\prime}\left(u_{j}\right) \rightarrow \theta, \text { in } L^{2} .
$$

If $\left\|u_{j}\right\|_{L^{2}} \rightarrow \infty$, we set $x_{j}=u_{j} /\left\|u_{j}\right\|_{L^{2}}$. Without loss of generality, we assume $x_{j} \rightarrow x_{0}$ in $L^{2}$, and hence $\Lambda^{-1} x_{j} \rightarrow \Lambda^{-1} x_{0}$ in $L^{2}$. For any $\delta \in(0,1)$ fixed, set

$$
\begin{aligned}
& C_{j}(t)= \begin{cases}\int_{0}^{1} N^{* \prime \prime}\left(t, \theta u_{j}(t)\right) d \theta & \text { if }\left|u_{j}(t)\right| \geq r_{1} / \delta, \\
\left(B_{1}(t)+\mu I_{2 n}\right)^{-1} & \text { otherwise, }\end{cases} \\
& \xi_{j}(t)=\int_{0}^{1} N^{* \prime \prime}\left(t, \theta u_{j}(t)\right) d \theta u_{j}(t)-C_{j}(t) u_{j}(t) .
\end{aligned}
$$

Then there exists a constant $M_{1}>0$ such that

$$
\left|\xi_{j}(t)\right| \leq M_{1} \quad \text { for a.e. } t \in(0,1),
$$


and

$$
(1-\delta)\left(B_{2}(t)+\mu I_{2 n}\right)^{-1}+\delta I_{2 n} \leq C_{j}(t) \leq(1-\delta)\left(B_{1}(t)+\mu I_{2 n}\right)^{-1}+\mu_{1}^{-1} \delta I_{2 n} .
$$

So for every $\epsilon>0$, there exists $\delta>0$ such that

$$
\left(\left(B_{2}(t)+\epsilon I_{2 n}\right)+\mu I_{2 n}\right)^{-1} \leq C_{j}(t) \leq\left(\left(B_{1}(t)-\epsilon I_{2 n}\right)+\mu I_{2 n}\right)^{-1}
$$

for all $t \in(0,1)$. Now we may further assume $C_{j}^{-1}(t) u(t) \rightarrow B_{0}(t) u(t)$ in $L^{2}$ for every $u \in L^{2}$ with $\mu I_{2 n}+B_{1}-\epsilon I_{2 n} \leq B_{0} \leq \mu I_{2 n}+B_{2}+\epsilon I_{2 n}$. Let $\Lambda^{-1} x_{0}(t)=y_{0}(t)$, from equations (3-22)-(3-24), we have

$$
J \dot{y}_{0}(t)+\left(B_{0}(t)-\mu I_{2 n}\right) y_{0}(t)=0, P y_{0}(0)=0=P y_{0}(1) .
$$

From assumption (1) and Proposition 2.13(3), for $\varepsilon>0$ is small enough, we have $v\left(B_{1}-\varepsilon I_{2 n}\right)=v\left(B_{2}+\varepsilon I_{2 n}\right)=0$ and $i\left(B_{1}-\varepsilon I_{2 n}\right)=i\left(B_{2}+\varepsilon I_{2 n}\right)$. So $v\left(B_{0}-\mu I_{2 n}\right)$ vanishes. This is impossible since $\left\|y_{0}\right\|_{L^{2}}=1$ and $y_{0}$ is a nontrivial solution of (3-25); thus $\left\|u_{j}\right\|_{L^{2}}$ is bounded. Assume $u_{j} \rightarrow u_{0}$ in $L^{2}$; then $\Lambda^{-1} u_{j} \rightarrow \Lambda^{-1} u_{0}$. Let $\zeta_{j}:=\Lambda^{-1} u_{j}+N^{* \prime}\left(t, u_{j}\right)$; then $N^{* \prime}\left(t, u_{j}\right)=\zeta_{j}-\Lambda^{-1} u_{j} \rightarrow-\Lambda^{-1} u_{0}$ in $L^{2}$, from (3-22). The Fenchel conjugate formula gives $u_{j}=N^{\prime}\left(\zeta_{j}-\Lambda^{-1} u_{j}\right) \rightarrow$ $N^{\prime}\left(-\Lambda^{-1} u_{0}\right)$ in $L^{2}$, by [Ekeland 1990, II, Theorem 4]. So $\psi$ satisfies the PS condition.

In order to continue the proof we need a lemma. Let $X$ be a Banach space and take $f \in C^{2}\left(X, R^{1}\right)$. Set $K=\left\{x \in X \mid f^{\prime}(x)=\theta\right\}$ and $f_{a}=\{x \in X \mid f(x) \leq a\}$. If $f^{\prime}(p)=\theta$ and $c=f(p)$, we say that $p$ is a critical point of $f$ and $c$ is a critical value. Otherwise, we say that $c \in \mathbb{R}$ is a regular value of $f$. For any $p \in K$, $f^{\prime \prime}(p)$ is a self-adjoint operator; the Morse index of $p$ is defined as the dimension of the negative space corresponding to the spectral decomposing, and is denoted by $m^{-}\left(f^{\prime \prime}(p)\right)$. We also set $m^{0}\left(f^{\prime \prime}\left(p_{0}\right)\right)=\operatorname{dim} \operatorname{ker} f^{\prime \prime}\left(p_{0}\right)$. If $f^{\prime \prime}(p)$ has a bounded inverse we say that $p$ is nondegenerate.

From [Chang 1993, Chapter III, Theorem 3.1; Chapter II, Theorems 5.1, 5.2 and Corollary 5.2], one can prove:

Lemma 3.9. Assume $f \in C^{2}(X, R)$ satisfies the PS condition, $f^{\prime}(\theta)=\theta$, and there is a positive integer $\gamma$ such that $\gamma \notin\left[m^{-}\left(f^{\prime \prime}(\theta)\right), m^{0}\left(f^{\prime \prime}(\theta)\right)+m^{-}\left(f^{\prime \prime}(\theta)\right)\right]$ and $H_{q}\left(X, f_{a} ; \mathbb{R}\right)=\delta_{q \gamma} \mathbb{R}$ for some regular value a $<f(\theta)$. Then $f$ has a critical point $p_{0} \neq \theta$ with $C_{\gamma}\left(f, p_{0}\right) \neq 0$. Moreover, if $\theta$ is a nondegenerate critical point and $m^{0}\left(f^{\prime \prime}\left(p_{0}\right)\right) \leq\left|\gamma-m^{-}\left(f^{\prime \prime}(\theta)\right)\right|$, then $f$ has another critical point $p_{1} \neq p_{0}, \theta$.

We resume the proof of the theorem. By (3-17), we have

$$
N^{* \prime \prime}(t, \theta)=\left(N^{\prime \prime}(t, \theta)\right)^{-1}=\left(H^{\prime \prime}(t, \theta)+\mu I_{2 n}\right)^{-1} .
$$


Thus, for any $u \in L^{2}$,

$$
\begin{aligned}
\left(\psi^{\prime \prime}(\theta) u, u\right) & =\int_{0}^{1}\left(\left(\Lambda^{-1} u, u\right)+\left(N^{* \prime \prime}(t, \theta) u, u\right)\right) d t \\
& =\int_{0}^{1}\left(\left(\Lambda^{-1} u, u\right)+\left(\left(B_{0}(t)+\mu I_{2 n}\right)^{-1} u(t), u(t)\right)\right) d t
\end{aligned}
$$

By definition, $m^{-}\left(\psi^{\prime \prime}(\theta)\right)=i_{\mu}\left(B_{0}\right)$ and $m^{0}\left(\psi^{\prime \prime}(\theta)\right)=v_{\mu}\left(B_{0}\right)$. By Propositions 2.10 and 2.13 , we have $i\left(B_{1}\right) \notin\left[i\left(B_{0}\right), i\left(B_{0}\right)+v\left(B_{0}\right)\right]$ if and only if $i_{\mu}\left(B_{1}\right) \in$ $\left[i_{\mu}\left(B_{0}\right), i_{\mu}\left(B_{0}\right)+v_{\mu}\left(B_{0}\right)\right]$; and $v\left(B_{0}\right)=0,\left|i\left(B_{0}\right)-i\left(B_{0}\right)\right| \geq n$ if and only if $v_{\mu}\left(B_{0}\right)=0,\left|i_{\mu}\left(B_{1}\right)-i_{\mu}\left(B_{0}\right)\right| \geq n$. Hence, by Lemma 3.9, we only need to show

$$
H_{q}\left(L^{2}, \psi_{-a} ; \mathbb{R}\right) \cong \delta_{q \gamma} \mathbb{R} \quad \text { for } q=0,1,2, \ldots,
$$

for $a>0$ is large enough, where $\gamma:=i_{\mu}\left(B_{1}\right)$. We proceed in three steps.

Step 1. For any $B_{1}, B_{2} \in \mathscr{L}_{2 n}^{\infty}$ with $B_{1}<B_{2}, i\left(B_{1}\right)=i\left(B_{2}\right)$ and $\nu\left(B_{2}\right)=0$, we have $L^{2}=E_{\mu}^{-}\left(B_{1}\right) \oplus E_{\mu}^{+}\left(B_{2}\right)$.

In fact, if $\theta \neq u \in E_{\mu}^{-}\left(B_{1}\right)$, then $\psi_{\left(\mu, B_{1}\right)}(u, u)<0$,

$$
\psi_{\left(\mu, B_{2}\right)}(u, u) \leq \psi_{\left(\mu, B_{1}\right)}(u, u)<0,
$$

and $u \notin E_{\mu}^{+}\left(B_{2}\right)$. So $E_{\mu}^{-}\left(B_{1}\right) \cap E_{\mu}^{+}\left(B_{2}\right)=\{\theta\}$. We only need to prove that $L^{2}=E_{\mu}^{-}\left(B_{1}\right)+E_{\mu}^{+}\left(B_{2}\right)$. By definition, $L^{2}=E_{\mu}^{-}\left(B_{2}\right) \oplus E_{\mu}^{+}\left(B_{2}\right)$, and $i_{\mu}\left(B_{2}\right)=$ $\operatorname{dim} E_{\mu}^{-}\left(B_{2}\right)<\infty$. Let $\left\{e_{j}\right\}_{j=1}^{\gamma}$ be a basis of $E_{\mu}^{-}\left(B_{1}\right)$ where $\gamma:=i_{\mu}\left(B_{1}\right)$. We have decompositions $e_{j}=e_{j}^{-}+e_{j}^{+}$with $e_{j}^{-} \in E_{\mu}^{-}\left(B_{2}\right)$ and $e_{j}^{+} \in E_{\mu}^{+}\left(B_{2}\right)$. If $\sum_{j=1}^{\gamma} \alpha_{j} e_{j}^{-}=0$, then $\bar{x}:=\sum_{j=1}^{\gamma} \alpha_{j} e_{j}=\sum_{j=1}^{\gamma} \alpha_{j} e_{j}^{+} \in E_{\mu}^{+}\left(B_{2}\right)$, and $\bar{x} \in E_{\mu}^{-}\left(B_{1}\right)$, so $\bar{x}=\theta$ and $\alpha_{j}=0, j=1,2, \ldots, \gamma$. Hence $\left\{e_{j}^{-}\right\}_{j=1}^{\gamma}$ is linear independent. Since $\operatorname{dim} E_{\mu}^{-}\left(B_{2}\right)=i_{\mu}\left(B_{2}\right)=i_{\mu}\left(B_{1}\right)=\gamma,\left\{e_{j}^{-}\right\}_{j=1}^{\gamma}$ is a basis of $E_{\mu}^{-}\left(B_{2}\right)$. For any $u \in L^{2}$ written as $u=u^{-}+u^{+}$with $u^{-} \in E_{\mu}^{-}\left(B_{2}\right)$ and $u^{+} \in E_{\mu}^{+}\left(B_{2}\right)$, we have $u^{-}=\sum_{j=1}^{\gamma} \beta_{j} e_{j}^{-}$. So $u=\sum_{j=1}^{\gamma} \beta_{j} e_{j}+\left(u^{+}-\sum_{j=1}^{\gamma} \beta_{j} e_{j}^{+}\right)$; the first sum lies in $E_{\mu}^{-}\left(B_{1}\right)$ and the remainder is in $E_{\mu}^{+}\left(B_{2}\right)$.

Step 2. For $\varepsilon>0$ small enough, set $M_{R}:=\left(E_{\mu}^{+}\left(B_{2}+\epsilon I_{2 n}\right) \cap B_{R}\right) \oplus E_{\mu}^{-}\left(B_{1}-\epsilon I_{2 n}\right)$. For $R, a>0$ large enough, then,

$$
H_{q}\left(L^{2}, \psi_{-a} ; \mathbb{R}\right)=H_{q}\left(M_{R}, M_{R} \cap \psi_{-a} ; \mathbb{R}\right) \quad \text { for } q=0,1,2, \ldots
$$

In fact, from assumption (1) and Proposition 2.13, we have $v\left(B_{2}+\varepsilon I_{2 n}\right)=0$ and $i\left(B_{2}+\varepsilon I_{2 n}\right)=i\left(B_{1}-\varepsilon I_{2 n}\right)$. In addition, by Step 1, we have

$$
L^{2}=E_{\mu}^{-}\left(B_{1}-\varepsilon I_{2 n}\right) \oplus E_{\mu}^{+}\left(B_{2}+\varepsilon I_{2 n}\right) .
$$


For every $u=u_{1}+u_{2} \in L^{2}$ with $u_{1} \in E_{\mu}^{-}\left(B_{1}-\epsilon I_{2 n}\right)$ and $u_{2} \in E_{\mu}^{+}\left(B_{2}+\epsilon I_{2 n}\right)$, from (3-21) we have

$$
\begin{aligned}
& \left(\psi^{\prime}(u), u_{2}-u_{1}\right) \\
& =\int_{0}^{1}\left(\left(\Lambda^{-1} u, u_{2}-u_{1}\right)+\left(N^{* \prime}(t,-u), u_{1}-u_{2}\right)\right) d t \\
& =-\int_{0}^{1}\left(\left(\Lambda^{-1} u_{1}, u_{1}\right)+\left(\int_{0}^{1} N^{* \prime \prime}(t,-\theta u) d \theta u_{1}, u_{1}\right)\right) d t \\
& +\int_{0}^{1}\left(\left(\Lambda^{-1} u_{2}, u_{2}\right)-\left(\int_{0}^{1} N^{* \prime \prime}(t,-\theta u) d \theta u_{2}, u_{2}\right)\right) d t \\
& \geq-\int_{0}^{1}\left(\left(\Lambda^{-1} u_{1}, u_{1}\right)+\left(\left(\mu I_{2 n}+B_{1}(t)-\varepsilon I_{2 n}\right)^{-1} u_{1}, u_{1}\right)\right) d t \\
& +\int_{0}^{1}\left(\left(\Lambda^{-1} u_{2}, u_{2}\right)+\left(\left(\mu I_{2 n}+\varepsilon I_{2 n}+B_{2}(t)\right)^{-1} u_{2}, u_{2}\right)\right) d t-c_{3},
\end{aligned}
$$

where $c_{3}>0$ is a constant. Now we bound these last two integrals using the fact that in the subspace $E_{\mu}^{-}\left(B_{1}-\varepsilon I_{2 n}\right)$ of $L^{2}$, the norm $\|\cdot\|_{L^{2}}$ is equivalent to $\|\cdot\|_{1}$ defined by

$$
\|u\|_{1}:=\left(-\int_{0}^{1}\left[\left(\Lambda^{-1} u, u\right)+\left(\left(\mu I_{2 n}+B_{1}(t)-\varepsilon I_{2 n}\right)^{-1} u, u\right)\right] d t\right)^{1 / 2}
$$

in this way we obtain

$$
\left(\psi^{\prime}(u), u_{2}-u_{1}\right) \geq c_{2}\left\|u_{2}\right\|_{L^{2}}^{2}+c_{1}\left\|u_{1}\right\|_{L^{2}}^{2}-c_{3},
$$

with $c_{1}, c_{2}>0$. Thus when $R$ is large enough we have $\left(\psi^{\prime}(u), u_{2}-u_{1}\right)>1$ for every $u=u_{1}+u_{2}$ with $u_{1} \in E_{\mu}^{-}\left(B_{1}-\epsilon I_{2 n}\right), u_{2} \in E_{\mu}^{+}\left(B_{2}+\epsilon I_{2 n}\right)$ and $\left\|u_{2}\right\|_{L^{2}} \geq R$, or $\left\|u_{1}\right\|_{L^{2}} \geq R$. For any $u=u_{2}+u_{1} \notin M_{R}$, let $\sigma(t, u)=e^{-t} u_{2}+e^{t} u_{1}, T_{u}=$ $\ln \left\|u_{2}\right\|-\ln R$, and

$$
\eta\left(t, u_{2}+u_{1}\right)= \begin{cases}u_{2}+u_{1} & \text { if }\left\|u_{2}\right\| \leq R, \\ \sigma\left(T_{u} t, u\right) & \text { if }\left\|u_{2}\right\|>R .\end{cases}
$$

Then $\eta:[0,1] \times L^{2} \rightarrow L^{2}$ is continuous and satisfies $\eta(0, \cdot)=\operatorname{id}_{L^{2}}, \eta\left(1, L^{2}\right) \subset M_{R}$, $\eta\left(1, \psi_{a}\right) \subset \mathcal{M}_{R} \cap \psi_{a}$,

$$
\eta\left(t, \psi_{a}\right) \subset \psi_{a},\left.\quad \eta(t, \cdot)\right|_{\mathcal{M}_{R}}=\operatorname{id}_{\mathcal{M}_{R}} \quad \text { for all } t \in[0,1] .
$$

So $\left(M_{R}, M_{R} \cap \psi_{a}\right)$ is a deformation retract of $\left(L^{2}, \psi_{a}\right)$, yielding (3-27).

Step 3. For $R,-a>0$ are large enough,

$$
H_{q}\left(\mu_{R}, \mu_{R} \cap \psi_{a} ; \mathbb{R}\right) \cong \delta_{q \gamma} \mathbb{R} \text { for } q=0,1, \ldots
$$


In fact, we have from (3-18) and (3-19) that

$$
\begin{aligned}
& \int_{0}^{1} N^{*}(t, u(t)) d t \\
& =\int_{0}^{1}\left(\int_{0}^{1} \theta d \theta \int_{0}^{1} N^{* \prime \prime}(t, \theta s u(t)) d s u(t), u(t)\right) d t+\int_{0}^{1} N^{*}(t, \theta) d t \\
& \quad \leq \int_{|u(t)| \geq k r_{1}}\left(\int_{0}^{1} \theta d \theta \int_{0}^{1} N^{* \prime \prime}(t, \theta s u(t)) d s u(t), u(t)\right) d t+M_{k} \\
& =\int_{|u(t)| \geq k r_{1}}\left(\iint_{|\theta s u(t)| \geq r_{1}, \theta, s \in[0,1]} \theta N^{* \prime \prime}(t, \theta s u(t)) d s d \theta u(t), u(t)\right) d t \\
& \quad+\int_{|u(t)| \geq k r_{1}}\left(\iint_{|\theta s u(t)| \leq r_{1}, \theta, s \in[0,1]} N^{* \prime \prime}(t, \theta s u(t)) d s d \theta u(t), u(t)\right) d t+M_{k} \\
& \leq \frac{1}{2} \int_{0}^{1}\left(\left(\mu I_{2 n}+B_{1}(t)\right)^{-1} u(t), u(t)\right) d t \\
& \quad+\mu_{1}^{-1} \int_{0}^{1}(u(t), u(t)) d t \iint_{|\theta s| \leq \frac{1}{k}, \theta, s \in[0,1]} \theta d s d \theta+M_{k},
\end{aligned}
$$

where $M_{k}$ is a constant depending only on $k$. Hence, for every $\varepsilon>0$ there exists a constant $M$ such that

$$
\int_{0}^{1} N^{*}(t, u(t)) d t \leq \frac{1}{2} \int_{0}^{1}\left(\left(\mu I_{2 n}+B_{1}(t)-\varepsilon I_{2 n}\right)^{-1} u(t), u(t)\right) d t+M
$$

for all $u \in L^{2}$. Together with (3-20), this yields, for any $u=u_{1}+u_{2}$ with $u_{1} \in$ $E_{\mu}^{-}\left(B_{1}-\epsilon I_{2 n}\right)$ and $u_{2} \in E_{\mu}^{+}\left(B_{2}+\epsilon I_{2 n}\right) \cap B_{R}$, the bound

$$
\psi(u) \leq-c_{1}\left\|u_{1}\right\|_{L^{2}}^{2}+c_{4}\left\|u_{1}\right\|_{L^{2}}+c_{5},
$$

where $c_{4}, c_{5}>0$, and $c_{1}$ is the constant in (3-28). Hence $\psi(u) \rightarrow-\infty$ if and only if $\left\|u_{1}\right\| \rightarrow+\infty$ uniformly in $u_{2} \in E_{\mu}^{+}\left(B_{2}+\epsilon I_{2 n}\right) \cap B_{R}$. Thus there exist $T>0$, $a_{1}<a_{2}<-T$, and $R_{0}>R_{1}>R_{2}>0$ such that

$$
\begin{aligned}
\left(E_{\mu}^{+}\left(B_{2}+\epsilon I_{2 n}\right)\right. & \left.\cap B_{R_{0}}\right) \oplus\left(E_{\mu}^{-}\left(B_{1}-\epsilon I_{2 n}\right) \backslash B_{R_{2}}\right) \subset \psi_{a_{1}} \cap \mu_{R_{0}} \\
& \subset\left(E_{\mu}^{+}\left(B_{2}+\epsilon I_{2 n}\right) \cap B_{R_{0}}\right) \oplus\left(E_{\mu}^{-}\left(B_{1}-\epsilon I_{2 n}\right) \backslash B_{R_{1}}\right) \subset \psi_{a_{2}} \cap M_{R_{0}} .
\end{aligned}
$$

For any $u \in M_{R_{0}} \cap\left(\psi_{a_{2}} \backslash \psi_{a_{1}}\right)$, since $\sigma(t, u)=e^{-t} u_{2}+e^{t} u_{1}$, the function $\psi(\sigma(t, x))$ is continuous in $t$ and satisfies $\psi(\sigma(0, x))=\psi(u)>a_{1}$ and $\psi(\sigma(t, u)) \rightarrow-\infty$ as $t \rightarrow+\infty$. Thus there exists a unique $t=T_{1}(u)$ such that $\psi(\sigma(t, u))=a_{1}$. Since

$$
\begin{aligned}
\frac{d}{d t} \psi(\sigma(t, u)) & =\left\langle d \psi(\sigma(t, u)), \sigma^{\prime}(t, u)\right\rangle \\
& =\left\langle d \psi\left(e^{-t} u_{2}+e^{t} u_{1}\right),-e^{-t} u_{2}+e^{t} u_{1}\right\rangle \leq-1
\end{aligned}
$$


as $t>0$, by the implicit function theorem, $t=T_{1}(u)$ is continuous. Define

$$
\eta_{1}(t, u)=u, u \in \psi_{a_{1}} \cap \mathcal{M}_{R_{0}}=\sigma\left(T_{1}(u) t, u\right), u \in \mathcal{M} \cap\left(\psi_{a_{2}} \backslash \psi_{a_{1}}\right) ;
$$

then $\eta_{1}:[0,1] \times \psi_{a_{2}} \cap \mathcal{M}_{R_{0}} \rightarrow \psi_{a_{2}} \cap \mu_{R_{0}}$ is a deformation from $\psi_{a_{2}} \cap \mathcal{M}_{R_{0}}$ to $\psi_{a_{1}} \cap \mu_{R_{0}}$. Set $\tau_{1}:=\eta_{1}(1, \cdot): \mu_{R_{0}} \cap \psi_{a_{2}} \rightarrow \mu_{R_{0}} \cap \psi_{a_{1}}$, and define

$$
\tau_{2}(u)= \begin{cases}u & \text { if }\left\|u_{1}\right\| \geq R_{1}, \\ u_{2}+\left(u_{1} /\left\|u_{1}\right\|\right) R_{1} & \text { if }\left\|u_{1}\right\|<R_{1} .\end{cases}
$$

Then $\tau=\tau_{2} \circ \tau_{1}$ is a strong deformation retract:

$$
\tau: M_{R_{0}} \cap \psi_{a_{2}} \rightarrow\left(E_{\mu}^{+}\left(B_{2}+\epsilon I_{2 n}\right) \cap B_{R_{0}}\right) \oplus\left(E_{\mu}^{-}\left(B_{1}-\epsilon I_{2 n}\right) \backslash \operatorname{int} B_{R_{1}}\right),
$$

where int $B_{R_{1}}$ is the interior of $B_{R_{1}}$. Hence, for $q=0,1,2, \ldots$,

$$
\begin{aligned}
H\left(\mu_{R_{0}}, \mu_{R_{0}} \cap \psi_{a_{2}} ; \mathbb{R}\right) \cong & H_{q}\left(\left(E_{\mu}^{+}\left(B_{2}+\epsilon I_{2 n}\right) \cap B_{R_{0}}\right) \oplus E_{\mu}^{-}\left(B_{1}-\epsilon I_{2 n}\right),\right. \\
& \left.\left(E_{\mu}^{+}\left(B_{2}+\epsilon I_{2 n}\right) \cap B_{R_{0}}\right) \oplus\left(E_{\mu}^{-}\left(B_{1}-\epsilon I_{2 n}\right) \backslash \operatorname{int} B_{R_{1}}\right) ; \mathbb{R}\right) \\
\cong & H_{q}\left(E_{\mu}^{-}\left(B_{1}-\epsilon I_{2 n}\right) \cap B_{R_{1}}, \partial\left(E_{\mu}^{-}\left(B_{1}-\epsilon I_{2 n}\right) \cap B_{R_{1}}\right) ; \mathbb{R}\right) \\
\cong & \delta_{q \gamma} \mathbb{R} .
\end{aligned}
$$

Remark. The method of the proof of (3-26) comes from [Chang 1993], but we have modified it to suit our case.

\section{Acknowledgements}

I would like to express my sincere thanks to Professor Yiming Long for his frequent valuable discussions with me. I also want to express my thanks to the referee for valuable comments on a revision of the manuscript.

\section{References}

[Chang 1986] K. C. Chang, Critical point theory and its application, Shanghai Sci. Tech. Press, Shanghai, 1986. In Chinese. MR 87m:58001 Zbl 0698.58002

[Chang 1993] K. C. Chang, Infinite-dimensional Morse theory and multiple solution problems, Prog. Nonlinear Diff. Eq. Appl. 6, Birkhäuser, Boston, 1993. MR 94e:58023 Zbl 0779.58005

[Chang et al. 1997] K. C. Chang, J. Q. Liu, and M. J. Liu, "Nontrivial periodic solutions for strong resonance Hamiltonian systems”, Ann. Inst. H. Poincaré Anal. Non Linéaire 14:1 (1997), 103-117. MR 97m:58166 Zbl 0881.34061

[Conley and Zehnder 1984] C. Conley and E. Zehnder, "Morse-type index theory for flows and periodic solutions for Hamiltonian equations", Comm. Pure Appl. Math. 37:2 (1984), 207-253. MR 86b:58021 Zbl 0559.58019

[Dong 2005] Y. Dong, "Index theory, nontrivial solutions, and asymptotically linear second-order Hamiltonian systems", J. Differential Equ. 214:2 (2005), 233-255. MR 2145250 Zbl 02189404 
[Dong and Long 1997] D. Dong and Y. Long, "The iteration formula of the Maslov-type index theory with applications to nonlinear Hamiltonian systems", Trans. Amer. Math. Soc. 349:7 (1997), 2619-2661. MR 97i:58028 Zbl 0870.58024

[Ekeland 1990] I. Ekeland, Convexity methods in Hamiltonian mechanics, Ergebnisse der Mathematik und ihrer Grenzgebiete (3) 19, Springer, Berlin, 1990. MR 91f:58027 Zbl 0707.70003

[Ekeland and Hofer 1985] I. Ekeland and H. Hofer, "Periodic solutions with prescribed minimal period for convex autonomous Hamiltonian systems", Invent. Math. 81:1 (1985), 155-188. MR 87b: $58028 \mathrm{Zbl} 0594.58035$

[Ekeland and Hofer 1987] I. Ekeland and H. Hofer, "Convex Hamiltonian energy surfaces and their periodic trajectories”, Comm. Math. Phys. 113:3 (1987), 419-469. MR 89c:58035 Zbl 0641.58038

[Fei 1995] G. H. Fei, "Relative Morse index and its application to Hamiltonian systems in the presence of symmetries", J. Differential Equ. 122:2 (1995), 302-315. MR 96i:58028 Zbl 0840.34032

[Fei and Qiu 1997] G. Fei and Q. Qiu, "Periodic solutions of asymptotically linear Hamiltonian systems", Chinese Ann. Math. Ser. B 18:3 (1997), 359-372. MR 98k:58044 Zbl 0884.58081

[Li and Liu 1989] S. J. Li and J. Q. Liu, "Morse theory and asymptotic linear Hamiltonian system", J. Differential Equ. 78:1 (1989), 53-73. MR 90d:58041 Zbl 0672.34037

[Liu et al. 2002] C. G. Liu, Y. Long, and C. Zhu, "Multiplicity of closed characteristics on symmetric convex hypersurfaces in $\mathbb{R}^{2 n}$ ", Math. Ann. 323 (2002), 201-215. MR 2003h:58013 Zbl 1005.37030

[Long 1990] Y. M. Long, "Maslov-type index, degenerate critical points, and asymptotically linear Hamiltonian systems”, Sci. China Ser. A 33:12 (1990), 1409-1419. MR 92d:58171 Zbl 0736.58022

[Long 1997] Y. Long, "A Maslov-type index theory for symplectic paths", Topol. Methods Nonlinear Anal. 10:1 (1997), 47-78. MR 99h:58031 Zbl 0977.53075

[Long 2002] Y. Long, Index theory for symplectic paths with applications, Progress in Math. 207, Birkhäuser, Basel, 2002. MR 2003d:37091 Zbl 1012.37012

[Long and Zehnder 1990] Y. M. Long and E. Zehnder, "Morse-theory for forced oscillations of asymptotically linear Hamiltonian systems", pp. 528-563 in Stochastic processes, physics and geometry (Ascona and Locarno, 1988), edited by S. Albeverio et al., World Sci. Publishing, Teaneck, NJ, 1990. MR 92j:58019 Zbl 0980.60501

[Long and Zhu 2000] Y. Long and C. Zhu, "Maslov-type index theory for symplectic paths and spectral flow. II”, Chinese Ann. Math. Ser. B 21:1 (2000), 89-108. MR 2001i:58049 Zbl 0959.58017

[Long and Zhu 2002] Y. Long and C. Zhu, "Closed characteristics on compact convex hypersurfaces in $\mathbb{R}^{2 n}$ ", Ann. of Math. (2) 155:2 (2002), 317-368. MR 2003e:37083 Zbl 1028.53003

[Su 1998] J. Su, "Nontrivial periodic solutions for the asymptotically linear Hamiltonian systems with resonance at infinity", J. Differential Equations 145:2 (1998), 252-273. MR 99b:58194 Zbl 0944.34034

[Zhu and Long 1999] C. Zhu and Y. Long, "Maslov-type index theory for symplectic paths and spectral flow. I", Chinese Ann. Math. Ser. B 20:4 (1999), 413-424. MR 2001i:58048 Zbl 0959.58016

Received January 26, 2004. Revised August 27, 2004.

\section{YUJUN DONG}

DEPARTMENT OF MATHEMATICS

NANJING NORMAL UNIVERSITY

NANJING, JiAngSU 210097, P. R. China

yjdong@eyou.com 